\title{
FACE: Fast AES CTR mode Encryption Techniques based on the Reuse of Repetitive Data
}

\author{
Jin Hyung Park and Dong Hoon Lee \\ Center for Information Security Technologies, \\ Korea University, Seoul, Republic of Korea \\ jhpark.embedsec.korea@gmail.com, donghlee@korea.ac.kr
}

\begin{abstract}
The Advanced Encryption Standard (AES) algorithm and Counter (CTR) mode are used for numerous services as an encryption technique that provides confidentiality. Even though the AES with counter (AES CTR) mode has an advantage in that it can process multiple data blocks in parallel, its implementation should also be observed to reduce the computational burden of current services.

In this paper, we propose an implementation method called FACE that can improve the performance of the AES CTR mode. The proposed method is based on five caches of frequently occurring intermediate values, so that it reduces the number of unnecessary computations. Our method can be employed in any AES CTR implementation, regardless of the platform, environment, or implementation method. There are two known AES implementation techniques, namely, counter-mode caching and bitslicing. FACE extends counter-mode caching in order to optimize the previous result and to maximize the scope of caching. We show that FACE can be applied efficiently to various implementations (table-based, bitsliced, and AES-NI-based). In particular, this is the first attempt to combine our extended counter-mode caching with bitsliced implementations of AES, and is also the first to apply counter-mode caching up to the round transformations of AES-NI implementation. To prove the efficiency of our proposed method, we conduct a performance evaluation in various environments, which we then compare with the previous fastest results. Our bitsliced FACE needs 6.41 cycles/byte on an Intel Core 2, and AES-NI-based FACE records 0.44 cycles/byte on an Intel Core i7.
\end{abstract}

Keywords: AES · counter mode · efficient software implementation · AES bitslicing · AES-NI

\section{Introduction}

The number of Internet users has increased rapidly with significant improvements in network technologies and services such as content delivery and VoIP have also emerged in response to demand. Because these Internet services are commonly based on usage-pricing models, service providers should consider a way to protect their service assets from illegal usage. Although there are several technologies for protecting either assets or information, most of these are based on providing confidentiality for their contents. On the other hand, the privacy of users should also be protected while they access the Internet. As a result, security services such as SSL are now widely adopted in various environments. The above-mentioned issues can be resolved using a cryptographic algorithm (e.g., AES, DES) for data confidentiality. However, adopting a cryptographic algorithm for current services is burdensome because it requires additional computational resources. Therefore, 
cryptographic algorithms must guarantee proven security and efficiency when employed in practical environments.

AES [NIS01a] and CTR mode [NIS01b] are used for numerous services (e.g., OMA DRM, VoIP, IPTV) as an encryption technique that preserves confidentiality. The AES CTR mode is not only operated as a standalone technique, but is also incorporated within authenticated encryption schemes, such as the AES GCM [NIS07] and AES CCM [NIS04]. Thus, optimizing the AES CTR mode results in improved performance not only for the AES CTR, but also the AES GCM and the AES CCM. While researches on improving the throughput of AES are ongoing, Intel has announced a set of instructions (AES-NI [Gue10]) that accelerate AES computations with dedicated hardware support. ARM also presents a set of instructions for accelerating AES on ARMv8 as crypto extension. However, AES-NI and Crypto Extension can be used only with specific processors. Many other processors (non-Intel, non-AMD, and pre-ARMv8-based) used in embedded devices or lightweight IoT devices do not yet support these instructions. This is the reason why enhancing the AES efficiency in software remains an important issue.

This study focuses on maximizing the efficiency of the AES algorithm using the counter mode. The AES CTR mode is one of the most widely used cryptographic algorithms for confidentiality, and is usually employed in seamless real-time services. The Open Mobile Appliance (OMA) Digital Right Management (DRM) v2.0 includes the AES CTR mode in PDCF format [All08] to protect streaming content such as music or video on mobile devices. Moreover, IPTV and VoIP services are high-profile Internet services that are usually based on the Secure Real-time Transfer Protocol (RTP) to protect data confidentiality. Even though Secure RTP improves security compared to previous versions of RTP, IETF has also selected the AES CTR mode with consideration for efficiency [BMN $\left.{ }^{+} 04\right]$. In general, AES CTR mode is widely adopted in many current applications for the following reasons. First, its security is proven in [McG02]. In addition, it has many advantages with respect to efficiency over AES with other operation modes, such as CBC. For example, the AES CTR mode can be processed in parallel, regardless of encryption or decryption. Moreover, it does not require the implementation of the AES decryption algorithm. When the services are deployed in lightweight devices that have relatively limited computational capabilities, it is even more important to conserve computation resources.

In this paper, we present an efficient implementation method for the AES CTR mode, called FACE (Fast AES CTR mode Encryption). FACE can be applied to existing implementation, regardless of platforms and implementation methods. The motive of our method can be summarized as follows. In the AES CTR mode, while increasing the number of blocks, the Initial Vector (IV) or counter that is used as an input value adds 1 to its least significant bit. The AES CTR mode encrypts sequentially increased IV rather than plaintext sequences. The output is XORed with the plaintext to produce a final ciphertext. We note that there are only small changes in each of the input blocks. The AES algorithm spreads the small variation in the input over the entire output by iterating its round transformation. This means that the front-located rounds operate a many-overlapped input in the AES CTR mode. If these overlapped values are cached and reused properly, the AES CTR mode can guarantee enhanced performance. Our proposed method, FACE, maximizes the scope for reuse in the AES CTR mode.

The contributions of our work are as follows.

1. We propose an efficient implementation technique for the CTR mode of AES. The proposed technique (FACE) extends the counter-mode caching that was first presented in [BS08] with credit to [Wu07]. Previous counter-mode caching technique only covered partial data of round transformation. Therefore, in order to show its efficiency, it has been applied only to table-based implementation. However, FACE can be employed in any AES CTR implementation, regardless of the platform, environment, or implementation method, as this technique can cover a round transformation 
entirely.

2. We show that FACE can be applied efficiently to existing implementation methods (e.g., table-based, bitsliced, and AES-NI-based implementations). In particular, our work is the first to combine counter-mode caching with bitsliced implementations of AES, and is also the first to apply counter-mode caching up to the round transformations of AES-NI implementation. Table-based FACE needs 12 instructions up to round 2, whereas the existing implementation [Pro] needs 128 instructions. Further, bitsliced FACE requires 74 instructions up to round 2, whereas [KS09] requires 618 instructions. AES-NI-based FACE requires 1 intrinsic instruction, 1 memory reference, and 1 arithmetic instruction up to round 2, whereas [Lib] requires 3 intrinsic instructions and 3 memory references. According to the Intel instruction latency and throughput [Cor18], a briefly calculated throughput (required cycle) for AES-NI-based FACE is 0.83 up to round 2, whereas for [Lib] it is 3.08.

3. Bitsliced FACE records 6.41 cycles/byte on an Intel Core 2 Q9550, and AES-NI-based FACE records 0.44 cycles/byte on an Intel Core i7 8700K. Our experimental results are recorded as the highest throughput ever achieved.

The rest of this paper is organized as follows. In section 2, we show related works involving attempts to enhance the performance of AES. In section 3, we briefly describe the AES and CTR mode of operation, as well as the relevant notations. In section 4, we present the techniques of FACE, which utilize repetitive data. In section 5, we explain our implementation and give the experimental results of FACE. Then, we compare our results to those of other software implementations. Section 6 discusses the possibility of cache-timing attacks. Finally, we conclude this paper in section 7.

\section{Related Work}

Attempts to improve the efficiency of the AES algorithm can be divided into two categories. One is to improve the implementation method of the hardware architecture, and another is to reduce the logic at the software level. The hardware approach has a limited advantage though, as it is comparatively less applicable than software.

Morioka and Satoh proposed AES implementation [MS04] that applies T-box, which is a combination of AES transformations (SubBytes, ShiftRows and MixColumns) [DR13]. Rouvroy et al. [RSQL04] suggest a design that combines the key schedule part and the data path part in a Xilinx FPGA. Sagib et al. [SRHDP03] propose a sequential architecture and pipeline architecture in FPGA, and Charot et al. [CYW03] implement a single round as a module in the Altera single-chip FPGA, enabling the degree of pipelining to be determined flexibly. Although there have been some efforts to improve the algorithm, most of them were based on a hardware implementation using FPGA. By applying loop unrolling and pipelining, several FPGA-based AES implementations have achieved a high throughput [GCVRSPGP10] $\left[\mathrm{QSH}^{+}\right.$09]. However, these hardware-based techniques have some restrictions, i.e. although hardware implementations commonly offer a high throughput compared to software designs, it is difficult to update built-in cryptographic modules - when the improved implementation were announced, if we want to apply this announced method, the update of implementation may not be as easy as software is - and these efforts cannot be applied to application software.

Another approach to enhancing the efficiency of AES can be found in improving the implementation logic in software. A widely known software implementation method is S-Box with pre-computation, as proposed by the authors of AES. It combines SubBytes, ShiftRows, and MixColumns, which are parts of the round operation of AES, and generates a pre-computation look-up table. Consequently, these three transformations can be replaced 
by a single lookup operation ${ }^{1}$. Bertoni et al. $\left[\mathrm{BBF}^{+} 02\right]$ report implementations for smaller CPUs by modifying the MixColumns transformation. Matsui and Fukuda [MF05] propose an efficient implementation that covers the Pentium III and Pentium 4. In addition, the most notable work is the AES implementation that uses the bitsliced method.

Matsui and Nakajima propose a bitslice AES implementation on an Intel Core 2, which is faster than any previous implementation [MN07]. The reported throughput of 9.2 cycles per byte is achieved only for a data chunk longer than 2,048 bytes. The bitsliced method works up to an enhanced performance as high as 7.59 cycles per byte on an Intel Core 2 Q9550 [KS09]. However, these works are not scalable because they can only operate on designated CPU architecture. Liu and Bass propose a parallel AES implementation [LB13] that achieves 70 cycles per block, although it requires a 164-core environment, which is not considered practical. On the other hand, techniques that optimize the number of calculations using the pre-computation method can be applied on various platforms regardless of their CPU architectures.

Bernstein and Schwabe [BS08] present a technique that improves the efficiency of the AES CTR mode, with credit to [Wu07]. Because of the property of the CTR mode, 15 bytes in the counter value of 16 bytes are maintained while processing 256 blocks, but only one byte changes. In [BS08], the calculation result is saved with the exception of the part influenced by the changed byte. The saved results are then reused while processing 256 blocks. This technique was first announced by Hongjun $\mathrm{Wu}$, and can be found in the Crypto ++ module [Lib] and in an open-source version of AES [Wu07]. In this paper, we maximize the reuse of intermediate values in the AES CTR mode. Our method improves [BS08, Lib, Wu07] and proposes novel techniques.

\section{Preliminaries}

In this section, we give a brief description of AES and CTR mode, as well as the relevant notation.

\subsection{Description of AES and CTR mode}

\subsubsection{Advanced Encryption Standard}

The Advanced Encryption Standard (AES)[NIS01a] is a symmetric key block cipher announced by the National Institute of Standards and Technology (NIST) to supersede DES. It has a fixed block size of 128 bits and supports key sizes of 128, 192, or 256 bits. In accordance with the key sizes, the AES algorithm is categorized into three types: AES-128, AES-192, and AES-256. Internally, AES uses the SPN structure, and repetitively performs a round function. The number of rounds is 10 for AES-128, 12 for AES-192, and 14 for AES-256. The round function is composed of four types of transformations: SubBytes, ShiftRows, MixColumns, and AddRoundKey. Each transformation operates on State, which is treated as a $4 \times 4$ matrix of bytes. The following briefly describes the four types of transformations of the AES round function.

SubBytes This operation substitutes one byte with another byte according to the S-Box table in the AES algorithm. In SubBytes, a byte calculation is not affected by other input bytes because the substitution deals with single bytes independently. Further, the same S-Box input always produces the same output, regardless of its position and rounds.

ShiftRows This transformation circularly transposes rows of State matrix from right to left. The amount of transposition is relevant to the row position. There are four rows in State, and the first row remains fixed. The bytes in the second row circularly change

\footnotetext{
${ }^{1}$ Some XOR operations still remain.
} 


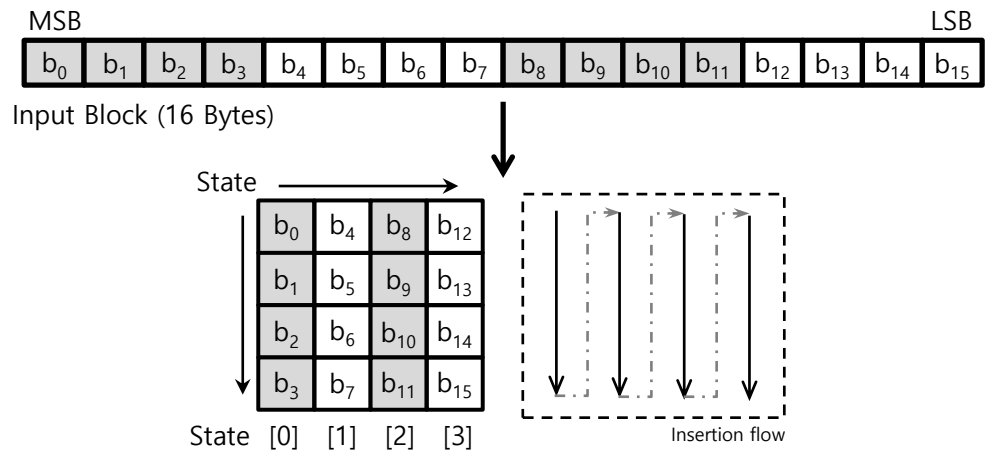

Figure 1: AES Block-to-State Transformation

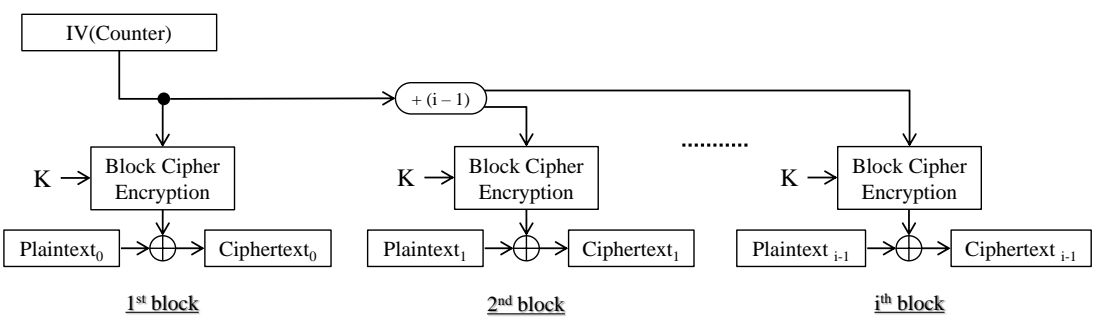

Figure 2: CTR Mode Encryption

their positions once, and the third and last rows change positions two and three times, respectively.

MixColumns While ShiftRows transforms bytes row-by-row, the MixColumns transformation is performed column-by-column. This operation combines the four bytes in each column. Each column of State matrix is changed into a new column using matrix multiplication with a constant matrix.

AddRoundKey This transformation simply XORs a given State with round keys. Each byte of State is XORed with the corresponding byte of the round key.

\subsubsection{AES Block-to-State Transformation}

To manipulate the internal input and output data, the AES algorithm uses State to form a series of data into a matrix. The AES data block can be expressed as a matrix of $1 \times 16$ bytes. State is 128 bits, but is expressed as a $4 \times 4$ matrix. At the beginning of the algorithm, the bytes in the input data block are inserted column-by-column into State, and from top to bottom in each column, as shown in Figure 1. State has four columns, which we refer to as State[0], State[1], State[2], and State[3], from left to right.

\subsubsection{Counter Mode}

In the case of counter mode, there is no need to implement a decryption algorithm of the block cipher. As shown in Figure 2, a 16 byte data block, called a counter, is encrypted by a block cipher in the place of plaintext. Then, this result and plaintext are merged by an XOR operation to create ciphertext. An $n$-bit counter is typically initialized to a pre-defined value (IV), and is then increased based on a pre-defined rule. The sequence of counter values must be distinguished from each other. In other words, the counter values must all have different values. 
Table 1: Notation

\begin{tabular}{|c|c|}
\hline Notation & Description \\
\hline \hline$X_{i, j}$ & $\begin{array}{c}\text { input (rin) or output (rout) State }\left(i^{\text {th }} \text { block, } j^{\text {th }} \text { round) }\right. \\
\text { (rin / rout is represented by } X)\end{array}$ \\
\hline$i n_{i}$ & $i^{\text {th }}$ byte of input data (counter value in CTR mode) block \\
\hline$r k_{i, j}$ & byte of round key (key for $i^{t h}$ round, $j^{t h}$ byte) \\
\hline$S_{i, j, k}$ & byte of State $\left(i^{\text {th }}\right.$ block, $j^{t h}$ round, $k^{t h}$ byte) \\
\hline$S[k]$ & $k^{t h}$ byte of input State \\
\hline$X_{i, j}[0]$ & $1^{\text {st }}$ column of $X_{i, j}$ \\
$X_{i, j}[1]$ & $2^{\text {nd }}$ column of $X_{i, j}$ \\
$X_{i, j}[2]$ & $3^{\text {rd }}$ column of $X_{i, j}$ \\
$X_{i, j}[3]$ & $4^{\text {th }}$ column of $X_{i, j}$ \\
\hline
\end{tabular}

\subsubsection{Bitslice Implementation}

The bitslice implementation technique was initially proposed by Biham to improve the software performance of DES [Bih97]. The bitslice technique simulates a hardware implementation in software, and all operations are expressed as a sequence of Boolean operations. On x86 processors, this technique is not practical for improving the performance of AES. However, on an x64 architecture, it is considered an interesting topic. A method to implement bitslice AES on x64 platforms was first reported in [Mat06]. Until then, 128-bit XMM registers were of no use, owing to their poor performance caused by treating a 128-bit instruction as two 64-bit operations on the processors (Pentium 4 and Athlon64). However, with Intel Core 2 processors, bitsliced AES is implemented to fully utilize XMM instructions [MN07], [KS09].

\subsection{Notation}

We use the notation described in Table 1 throughout the paper. We denote $X_{i, j}$ as the input or output State of the $j^{\text {th }}$ round of the $i^{\text {th }}$ block. For example, rin $n_{3,1}$ denotes the input State of the first round in the fourth block. This round function produces rout t $_{3,1}$. The $i^{\text {th }}$ byte of the input data block is denoted by $i n_{i}$ and $r k_{i, j}$ refers to the $j^{\text {th }}$ byte of the round key corresponding to the $i^{t h}$ round. A single byte of State is represented by $S[k]$. Because the block size of AES is 16 bytes, S[0] indicates the most significant byte (MSB) and S[15] indicates the least significant byte (LSB) of State. As mentioned above, $X_{i, j}[i]$ denotes the $i^{\text {th }}$ column of the input or output State from left to right. According to the AES block-to-state transformation, $X_{i, j}[i]$ is comprised of $\mathrm{S}[i * 4], \mathrm{S}[i * 4+1], \mathrm{S}[i * 4+2]$, and $\mathrm{S}[i * 4+3]$, where $i=0,1,2,3$. We define $S_{i, j, k}$ as the single byte of the particular State that indicates the $k^{\text {th }}$ byte of the $j^{\text {th }}$ round of the $i^{\text {th }}$ block.

\section{Implementation Technique Using Repetitive Data: FACE}

In this section, we present our implementation techniques to enhance the efficiency of AES CTR mode encryption. We reuse the repetitive partial data contained in the output State or intermediate calculation results of the round function, from round 0 (i.e. initial whitening. We denoted the initial whitening as 'round 0' for generalizing the naming rules of targets) to round 2 . In this paper, we present five types of reuse techniques, which are briefly described below.

- $\mathrm{FACE}_{r d 0}$ : This technique caches the result-State of an initial whitening (round 0) and reuses the cached data at the next block. While the overlapping area of the 
result-State is 15 bytes, we cache and reuse only 12 bytes of the result-State to minimize the update frequency of the cache. Section 4.1 explains this technique and its benefit.

- $\mathrm{FACE}_{r d 1}$ : This phase was already introduced in [BS08, Lib, Wu07]. We denote this technique as $\mathrm{FACE}_{r d 1}$ and describe it in Section 4.2.

- $\mathrm{FACE}_{r d 1+}$ : In AES round 1, FACE ${ }_{r d 1}$ caches and reuses 12 bytes of the result-State. Originally, the remaining 4 bytes should be recalculated in every block. $\mathrm{FACE}_{r d 1+}$ is a technique that generates pre-computation values for these 4 bytes of the result-State, which are not covered by $\mathrm{FACE}_{r d 1}$. These values can be generated either before or during encryption. Section 4.3 explains this technique.

- $\mathrm{FACE}_{r d 2}$ : This technique caches and reuses 16 bytes of State after the MixColumns() and AddRoundKey() transformations in round 2. Our implementation saves 16 instructions ( 8 integer +8 load) more as compared with [BS08, Lib, Wu07]. Section 4.4 gives a detailed explanation of this technique.

- $\mathrm{FACE}_{r d 2+}$ : In AES round 2, $\mathrm{FACE}_{r d 2}$ caches and reuses 16 bytes of the intermediate calculation result. Similar to round 1, the remaining part should be recalculated in every block. Then, this calculated remaining part and the cached data of $\mathrm{FACE}_{r d 2}$ are merged by XOR operations to complete round 2. FACE $r d 2+$ generates precomputation values for this remaining calculation result, which is not covered by FACE $_{r d 2}$. These values also can be generated either before or during encryption. Section 4.5 accounts for this technique.

Existing counter-mode caching method only just deals with partial result of each round transformation. It is suitable only for table-based implementation to show its efficiency because the remaining result should be recalculated in every block. However, FACE can cover a round transformation entirely. FACE can be applied efficiently to existing implementation methods (table-based and bitsliced) and even to AES-NI.

We elucidate our methods using the general AES model in order to represent each method convincingly.

\subsection{Technique Applied to Initial Whitening (FACE $\left.{ }_{r d 0}\right)$}

The input value of the first block is initialized to a pre-defined value (i.e., IV) in the AES CTR mode. While processing multiple blocks of plaintext, the value of IV increases, but changes only the last byte of IV in most cases. This means that the only difference between a given block and the previous block is the last byte, unless the last byte exceeds $0 \mathrm{xFF}$. For example, if $\mathrm{IV}_{0}$ (the input value of the first block) is initialized to $\{0 \mathrm{x} 00000000$, 0x00000000, 0x00000000, 0x00000001\}, IV $\mathrm{IV}_{0}$ and $\mathrm{IV}_{1}$ (the input value of the next block, which is increased by the counter) have the same value, except in last 1 byte, as in the example below.

- $I V_{0}: 0 \times 000000000 \times 000000000 \times 000000000 \times 00000001$

. $\mathrm{IV}_{1}$ : $0 \times 000000000 \times 000000000 \times 000000000 \times 00000002$

In this case, most bytes do not change until the value of the last byte reaches $0 \mathrm{xFF}$. We use this property to reuse the result of the operation in initial whitening (round 0). The process of the initial whitening is as follows:

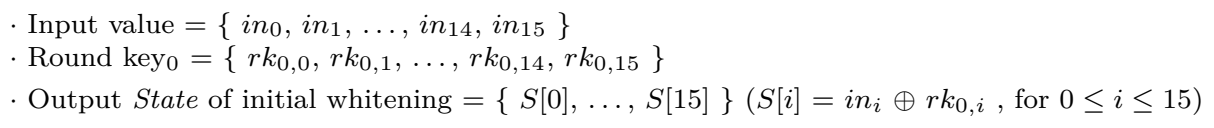




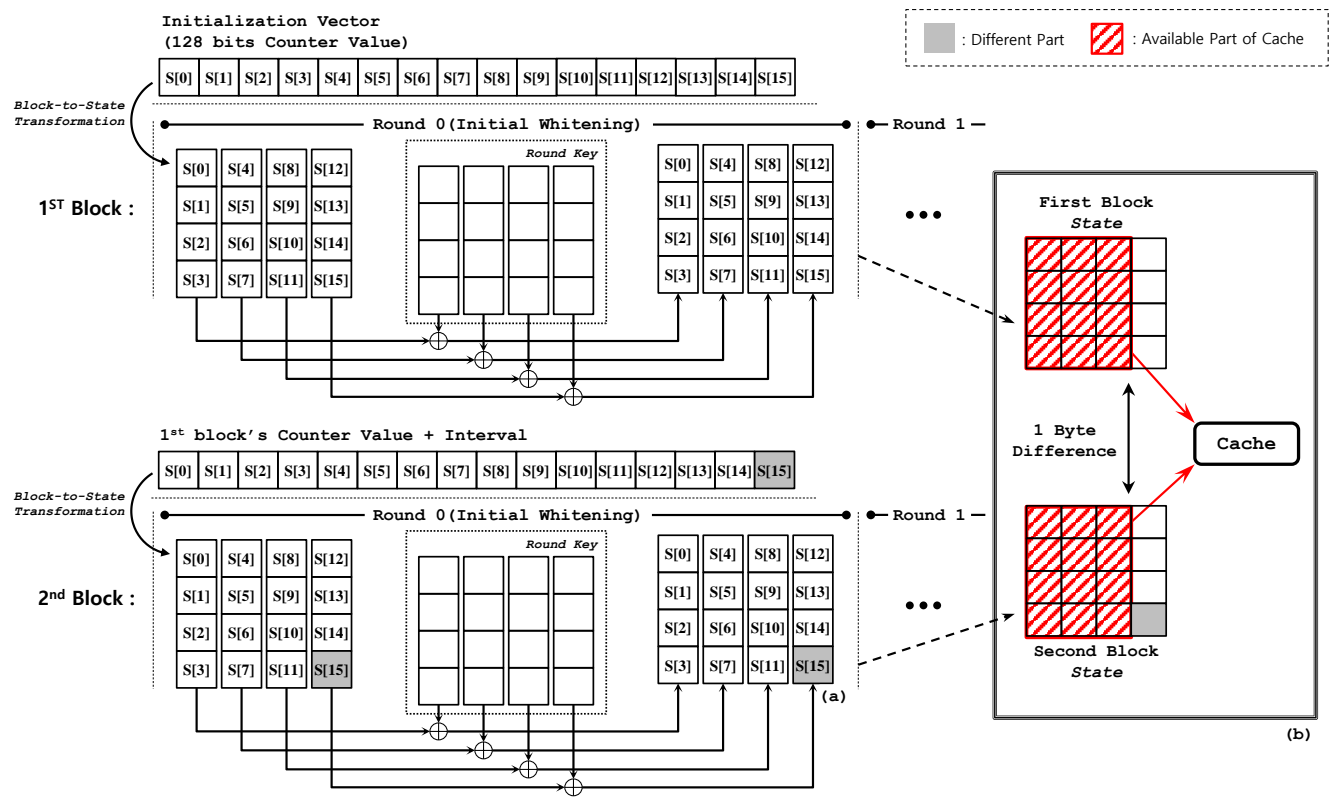

Figure 3: $\mathrm{FACE}_{r d 0}$ : AES initial whitening (round 0) of the first block and the second block in CTR mode. (a) shows the 1 byte difference between rout i, $_{0}$ and rout $t_{i+1,0}$. (b) indicates the cached part used by $\mathrm{FACE}_{r d 0}$.

In keeping with the above procedure, the following presents the process for the first block and the second block in the initial whitening (round 0 ).

First block input $\left(\mathrm{IV}_{0}\right)=\left\{i n_{0}, i n_{1}, \ldots, i n_{14}, i n_{15}\right\}$

. Second block input $\left(\mathrm{IV}_{1}\right)=\left\{i n_{0}, i n_{1}, \ldots, i n_{14}, i n_{15}^{\prime}\right\}$

- Round key $_{0}=\left\{r k_{0,0}, r k_{0,1}, r k_{0,2}, \ldots, r k_{0,14}, r k_{0,15}\right\}$

- Initial whitening output State of first block $\left(\operatorname{IV}_{0} \oplus \operatorname{Round}_{\text {key }}\right)=\{S[0], S[1], \ldots, S[14], S[15]\}$

- Initial whitening output State of second block $\left(\operatorname{IV}_{1} \oplus\right.$ Round keyo $)=\left\{S[0], S[1], \ldots, S[14], S^{\prime}[15]\right\}$

From results such as $I V_{0}$ and $I V_{1}$, we find that the first block output and second block output differ in the last 1 byte $\left(S[15]\right.$ and $\left.S^{\prime}[15]\right)$. FACE ${ }_{r d 0}$ reuses only rout $_{i, 0}[0]$, rout ${ }_{i, 0}[1]$, and rout $_{i, 0}[2]$ to minimize cache updating. Therefore, while operating $2^{22}-1$ successive blocks, ours does not need to update the cache information. Figure 3 indicates the process for the initial whitening (round 0) and the part of State cached from the round operation result. $\mathrm{FACE}_{r d 0}$ can use the same cached information until the last four bytes of IV reach OxFFFFFFFF after which the cache of FACE $_{r d 0}$ should be updated. For example, if the counter is increased by 1 and the IV value is 0 , the block that causes a carry at the $11^{\text {th }}$ byte of the counter value is the $4,294,967,297^{\text {th }}$ block. In this case, the cached information can be used while calculating 4,294,967,295 blocks instead of performing XOR operations. That is, $\mathrm{FACE}_{r d 0}$ updates the cached data only once throughout the $65.5 \mathrm{~GB}$ of plaintext.

\subsection{Technique Applied to Round $1\left(\mathrm{FACE}_{r d 1}\right)$}

The output State of the initial whitening (round 0) is used as the input of round 1. In round 1, $\mathrm{FACE}_{r d 1}$ can also cache and reuse data. Figure 4 depicts $\mathrm{FACE}_{r d 1}$. The feature of $\mathrm{FACE}_{r d 1}$ is that $\operatorname{rin}_{0,1}$ and $\operatorname{rin}_{1,1}$ are different only in the last 1 byte, as shown below.

\footnotetext{
- rout $_{i, 0}=\operatorname{rin}_{i, 1}$

- Comparison between $\operatorname{rin}_{0,1}$ and $\operatorname{rin}_{1,1}$$$
S_{0,1, k}=S_{1,1, k}, \text { for } 0 \leq k \leq 14
$$ 


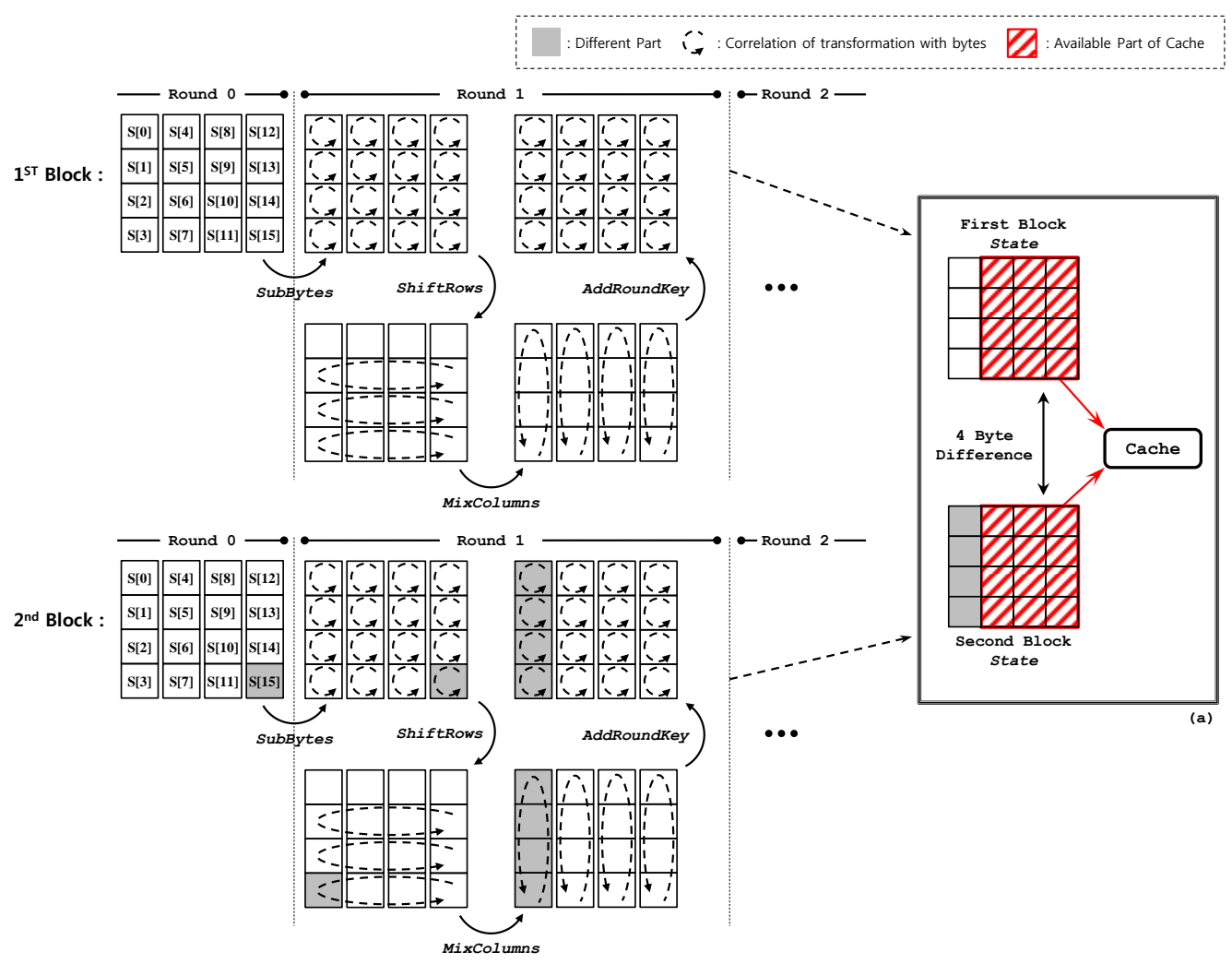

Figure 4: $\mathrm{FACE}_{r d 1}$ : The process of AES round 1 and the diffusion of the difference between the first block and the second block. (a) represents the cached part used by $\mathrm{FACE}_{r d 1}$.

$$
\cdot S_{0,1, k} \neq S_{1,1, k}, \text { if } k=15
$$

In round 1 , the difference in the last 1 byte of the input State affects only rout ${ }_{i, 1}[0]$ instead of affecting the whole State. Therefore, as shown in Figure 4, FACE ${ }_{r d 1}$ can cache and reuse the result of round 1 except for rout $_{i, 1}[0]$. The following presents the process of the first block and the second block in round 1 .

$$
\begin{aligned}
& \cdot \operatorname{rin}_{0,1}=\left\{S_{0,1,0}, S_{0,1,1}, \ldots, S_{0,1,14}, S_{0,1,15}\right\} \\
& \cdot \operatorname{rin}_{1,1}=\left\{S_{0,1,0}, S_{0,1,1}, \ldots, S_{0,1,14}, S_{1,1,15}\right\}
\end{aligned}
$$

are changed by four transformations to

$$
\begin{aligned}
& \text {. } \text { rout }_{0,1}=\left\{S_{0,1,0}^{\prime}, S_{0,1,5}^{\prime}, S_{0,1,10}^{\prime}, S_{0,1,15}^{\prime}\right. \text {, } \\
& S_{0,1,4}^{\prime}, S_{0,1,9}^{\prime}, S_{0,1,14}^{\prime}, S_{0,1,3}^{\prime}, \\
& S_{0,1,8}^{\prime}, S_{0,1,13}^{\prime}, S_{0,1,2}^{\prime}, S_{0,1,7}^{\prime} \text {, } \\
& \left.S_{0,1,12}^{\prime}, S_{0,1,1}^{\prime}, S_{0,1,6}^{\prime}, S_{0,1,11}^{\prime}\right\} \\
& \text { - } \text { rout }_{1,1}=\left\{S_{1,1,0}^{\prime}, S_{1,1,5}^{\prime}, S_{1,1,10}^{\prime}, S_{1,1,15}^{\prime}\right. \text {, } \\
& S_{0,1,4}^{\prime}, S_{0,1,9}^{\prime}, S_{0,1,14}^{\prime}, S_{0,1,3}^{\prime}, \\
& S_{0,1,8}^{\prime}, S_{0,1,13}^{\prime}, S_{0,1,2}^{\prime}, S_{0,1,7}^{\prime}, \\
& \left.S_{0,1,12}^{\prime}, S_{0,1,1}^{\prime}, S_{0,1,6}^{\prime}, S_{0,1,11}^{\prime}\right\}
\end{aligned}
$$

- Comparison between rout $_{0,1}$ and rout $_{1,1}$

$$
\text { - } \text { rout }_{0,1}[\mathrm{i}] \neq \text { rout }_{1,1}[\mathrm{i}] \text {, if } i=0
$$

$\cdot$ rout $_{0,1}[\mathrm{i}]=$ rout $_{1,1}[\mathrm{i}]$, for $1 \leq i \leq 3$ 


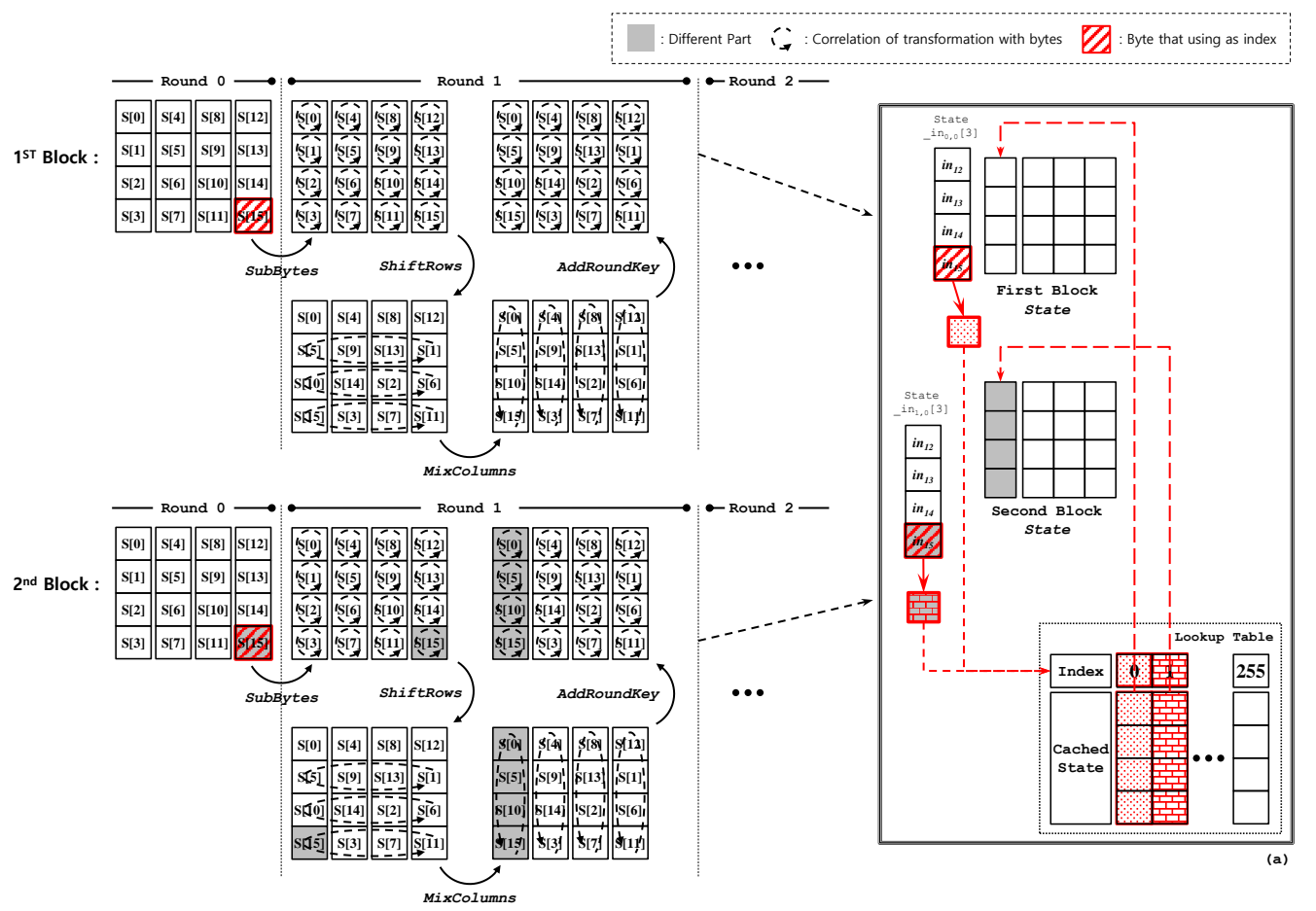

Figure 5: $\mathrm{FACE}_{r d 1+}$ : The process of AES round 1 and $\mathrm{FACE}_{r d 1+}$. (a) shows how to refer the pre-computation values.

Thus, we can cache rout $_{0,1}[\mathrm{i}](1 \leq i \leq 3)$ and reuse them for the next block. Because rout $_{i, 1}[1]$ is changed when $S[14]$ of $\operatorname{rin}_{i, 1}$ is altered, FACE $r d 1$ updates the cached data once for every $256\left(2^{8}\right)$ blocks.

\subsection{Additional Technique Applied to Round $1\left(\mathrm{FACE}_{r d 1+}\right)$}

In general, the implemented cryptographic algorithm in a real environment comprises two phases. The first phase is an initialization stage. In this stage, the cryptographic algorithms accept cryptographic parameters such as a secret-key and IV. In the case of AES, the secret-key is expanded to the round key as the AES key length, and IV is used as a counter in CTR mode. The second phase is the encryption/decryption stage. Input data are encrypted or decrypted using a fixed round key and IV. Once the cryptographic parameters are initialized, phase 2 can perform encryption or decryption processes using the same parameters. In many real environments, phase 1 is called only once, but phase 2 is performed several times.

In this section, we propose a technique that generates pre-computation values for rout $_{i, 1}[0]$ in phase 1 that are reused for round 1 in phase 2 ; if the length of the input data exceeds 256 blocks, pre-computation values can be generated and reused in phase 2 . As mentioned in Section 4.2, most of the result-State of round 1 (rout $_{i, 1}[1]$, rout ti, $_{1}[2]$, and rout $\left._{i, 1}[3]\right)$ are covered by $\mathrm{FACE}_{r d 1}$. However, rout $_{i, 1}[0]$ is changed for every block because the alteration of $i n_{15}$ affects all bytes of rout $_{i, 1}[0]$. Figure 7 shows the process of the State transformation in round 1 while the AES CTR mode operates. As shown in Figure 7, the factors that determine rout $_{i, 1}[0]$ are $S[0], S[5], S[10]$, and $S[15]$ of rin $_{i, 1}$. As already shown, S[15] of $r i n_{i, 1}$ is $i n_{15} \oplus r k_{0,15}$. Because the round key remains the same while working, S[15] of $\operatorname{rin}_{i, 1}$ is determined by $i n_{15}$. According to the increasing rule that adds 


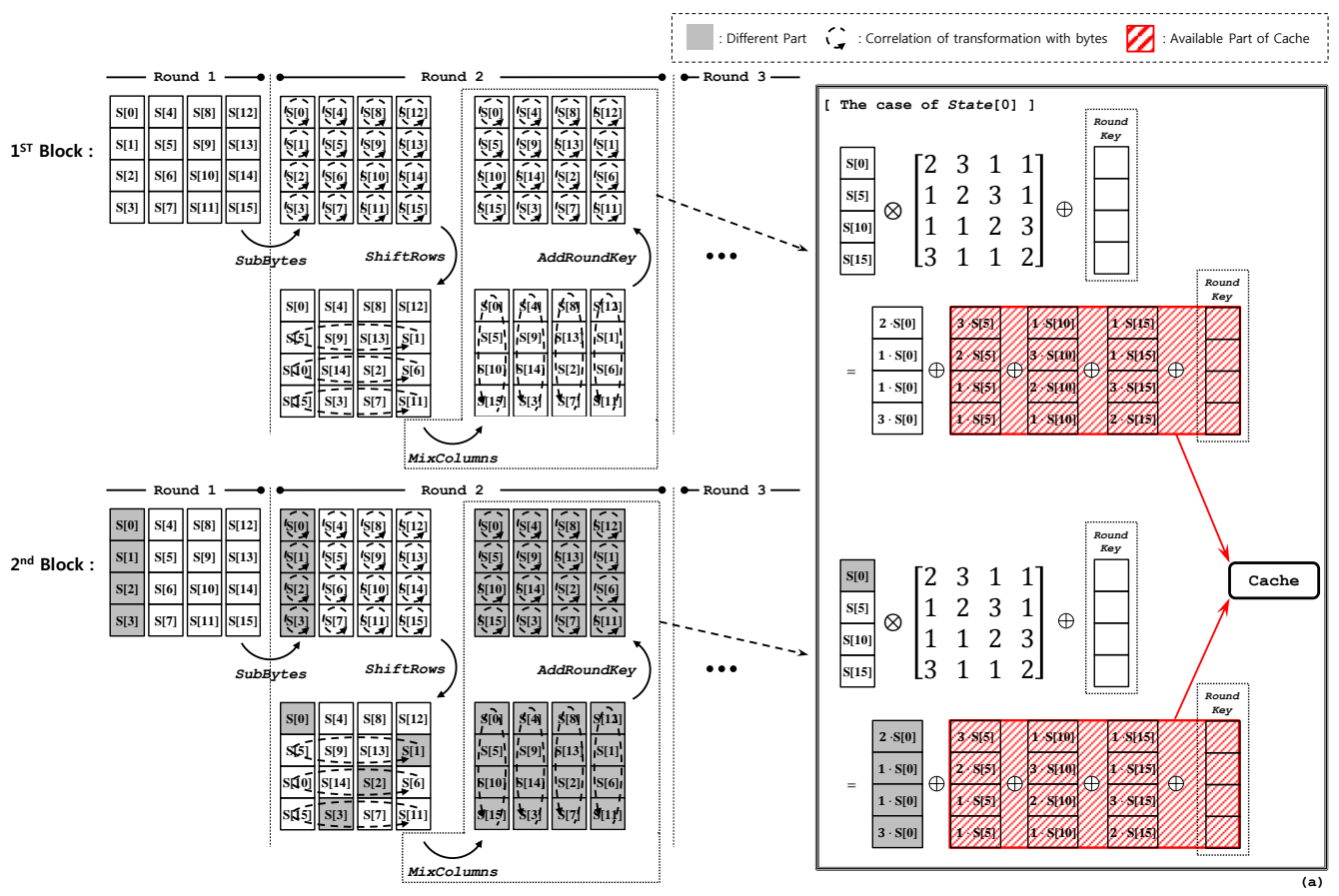

Figure 6: $\mathrm{FACE}_{r d 2}$ : The diffusion process of the difference between the first block and the second block in round 2. (a) indicates the MixColumns() and AddRoundKey() transformations using State[0]. It also shows the available part of the cache.

one to the previous counter value, $i n_{15}$ is changed continuously. However, $i n_{10}$ is altered only when the value of $i n_{11}-i n_{15}$ exceeds OxFFFFFFFFF. This means that $i n_{0}, i n_{5}$, and $i n_{10}$ are never changed while processing 1,099,511,627,776 blocks (16 TB). Thus, we can generate a temporary look-up table that uses $i_{15}$ as an index. If we create a table that determines rout $_{i, 1}[0]$, we need an additional $1 \mathrm{~KB}(4 \times 256)$ of memory because rout $_{i, 1}[0]$ is 4 bytes and $i n_{15}$ changes from $0 \times 00$ to $0 \mathrm{xFF}$. Although this table is not the same for every crypto instance, it can be pre-computed and determined in the initialization stage because it depends on the secret-key and IV. The initialization stage is performed once every instance, so we can improve the efficiency in the encryption/decryption stage using $\mathrm{FACE}_{r d 1+}$.

Pre-computation values can be used until the change of $i n_{15}$ influences $i n_{10}$, which occurs when $i n_{10}$ is changed on the next block by $\left[i n_{11}-i n_{15}\right]$ becoming [0xFFFFFFFFFF]. At this time, the pre-computation values must be updated. Updated values can be used for processing $2^{40}$ blocks without the need for an additional updating process. Thus, the efficiency of the calculation is improved.

\subsection{Technique Applied to Round $2\left(\mathrm{FACE}_{r d 2}\right)$}

In round 2, the result-State of round 1 is used as the input of round 2. Now, the difference in the number of bytes between $\operatorname{rin}_{0,2}$ and $\operatorname{rin}_{1,2}$ is four.

$$
\begin{aligned}
& \cdot \operatorname{rin}_{0,2}[\mathrm{i}] \neq \operatorname{rin}_{1,2}[\mathrm{i}], \text { if } i=0 \\
& \cdot \operatorname{rin}_{0,2}[\mathrm{i}]=\operatorname{rin}_{1,2}[\mathrm{i}], \text { for } 1 \leq i \leq 3
\end{aligned}
$$

Figure 6 describes the round 2 operation for the first and second blocks. At the end of round 2, the difference in State between $\operatorname{rin}_{0,2}$ and $\operatorname{rin}_{1,2}$ affects the whole byte of the 
result-State of round 2. Therefore, rout $_{0,2}$ and rout $_{1,2}$ are completely different. However, as in the other preceding techniques, we can also cache intermediate information in round 2 .

The following expresses the MixColumns() and AddRoundKey() transformations using State [0] as an example. Before the MixColumns () transformation, State[0] is comprised of $\{S[0], S[5], S[10]$, and $S[15]\}$ due to the ShiftRows() transformation.

$$
\begin{aligned}
\left(\begin{array}{c}
S^{\prime}[0] \\
S^{\prime}[5] \\
S^{\prime}[10] \\
S^{\prime}[15]
\end{array}\right)= & \left(\begin{array}{llll}
2 & 3 & 1 & 1 \\
1 & 2 & 3 & 1 \\
1 & 1 & 2 & 3 \\
3 & 1 & 1 & 2
\end{array}\right) \otimes\left(\begin{array}{c}
S[0] \\
S[5] \\
S[10] \\
S[15]
\end{array}\right) \oplus\left(\begin{array}{l}
r k_{2,0} \\
r k_{2,1} \\
r k_{2,2} \\
r k_{2,3}
\end{array}\right) \\
= & \left(\begin{array}{l}
2 \cdot S[0] \oplus 3 \cdot S[5] \oplus 1 \cdot S[10] \oplus 1 \cdot S[15] \oplus r k_{2,0} \\
1 \cdot S[0] \oplus 2 \cdot S[5] \oplus 3 \cdot S[10] \oplus 1 \cdot S[15] \oplus r k_{2,1} \\
1 \cdot S[0] \oplus 1 \cdot S[5] \oplus 2 \cdot S[10] \oplus 3 \cdot S[15] \oplus r k_{2,2} \\
3 \cdot S[0] \oplus 1 \cdot S[5] \oplus 1 \cdot S[10] \oplus 2 \cdot S[15] \oplus r k_{2,3}
\end{array}\right)
\end{aligned}
$$

Upon processing the MixColumns() transformation, as shown in Figure 6(a), the data related to $S[0]$ would change in rout $_{i, 2}[0]$, but the other data do not. Therefore, we can cache the intermediate calculation result for rout $_{i, 2}[0]$ as follows:

$$
\text { - Cached part of } \text { rout }_{i, 2}[0]:\left(\begin{array}{l}
3 \cdot S[5] \oplus 1 \cdot S[10] \oplus 1 \cdot S[15] \oplus r k_{2,0} \\
2 \cdot S[5] \oplus 3 \cdot S[10] \oplus 1 \cdot S[15] \oplus r k_{2,1} \\
1 \cdot S[5] \oplus 2 \cdot S[10] \oplus 3 \cdot S[15] \oplus r k_{2,2} \\
1 \cdot S[5] \oplus 1 \cdot S[10] \oplus 2 \cdot S[15] \oplus r k_{2,3}
\end{array}\right)
$$

This technique can be expanded to State[1], State[2] and State[3]. That is, State[1], State[2], and State[3] have changed parts that are related to $S[3], S[2]$, and $S[1]$ respectively. Thus $\mathrm{FACE}_{r d 2}$ caches the rest of the intermediate information, as follows.

$$
\begin{aligned}
& \text { - In the case of State[1], cached part of } \text { rout }_{i, 2}[1]:\left(\begin{array}{c}
2 \cdot S[4] \oplus 3 \cdot S[9] \oplus 1 \cdot S[14] \oplus r k_{2,4} \\
1 \cdot S[4] \oplus 2 \cdot S[9] \oplus 3 \cdot S[14] \oplus r k_{2,5} \\
1 \cdot S[4] \oplus 1 \cdot S[9] \oplus 2 \cdot S[14] \oplus r k_{2,6} \\
3 \cdot S[4] \oplus 1 \cdot S[9] \oplus 1 \cdot S[14] \oplus r k_{2,7}
\end{array}\right) \\
& \text { - In the case of State }[2] \text {, cached part of } \operatorname{rout}_{i, 2}[2]:\left(\begin{array}{c}
2 \cdot S[8] \oplus 3 \cdot S[13] \oplus 1 \cdot S[7] \oplus r k_{2,8} \\
1 \cdot S[8] \oplus 2 \cdot S[13] \oplus 1 \cdot S[7] \oplus r k_{2,9} \\
1 \cdot S[8] \oplus 1 \cdot S[13] \oplus 3 \cdot S[7] \oplus r k_{2,10} \\
3 \cdot S[8] \oplus 1 \cdot S[13] \oplus 2 \cdot S[7] \oplus r k_{2,11}
\end{array}\right) \\
& \text { - In the case of State[3], cached part of } \operatorname{rout}_{i, 2}[3]:\left(\begin{array}{c}
2 \cdot S[12] \oplus 1 \cdot S[6] \oplus 1 \cdot S[11] \oplus r k_{2,12} \\
1 \cdot S[12] \oplus 3 \cdot S[6] \oplus 1 \cdot S[11] \oplus r k_{2,13} \\
1 \cdot S[12] \oplus 2 \cdot S[6] \oplus 3 \cdot S[11] \oplus r k_{2,14} \\
3 \cdot S[12] \oplus 1 \cdot S[6] \oplus 2 \cdot S[11] \oplus r k_{2,15}
\end{array}\right)
\end{aligned}
$$

FACE $_{r d 2}$ can reuse these cached data while processing $255\left(2^{8}-1\right)$ consecutive blocks. The frequency of updates is equal to that of $\mathrm{FACE}_{r d 1}$ because the alteration of rin $_{i, 2}[1]$ affects the cached data.

\subsection{Additional Technique Applied to Round 2 (FACE ${ }_{r d 2+}$ )}

In this section, we propose a technique that generates pre-computation values for the remaining intermediate data, which is not covered by $F A C E_{r d 2}$. As we mentioned in section 4.3 , the pre-computation values for $\mathrm{FACE}_{r d 2+}$ can also be generated either before or during encryption (in phase 1 or phase 2 , respectively).

As described in section 4.4, rout $_{i, 2}[0]$ is calculated after the SubBytes () and ShiftRows() transformations as : 


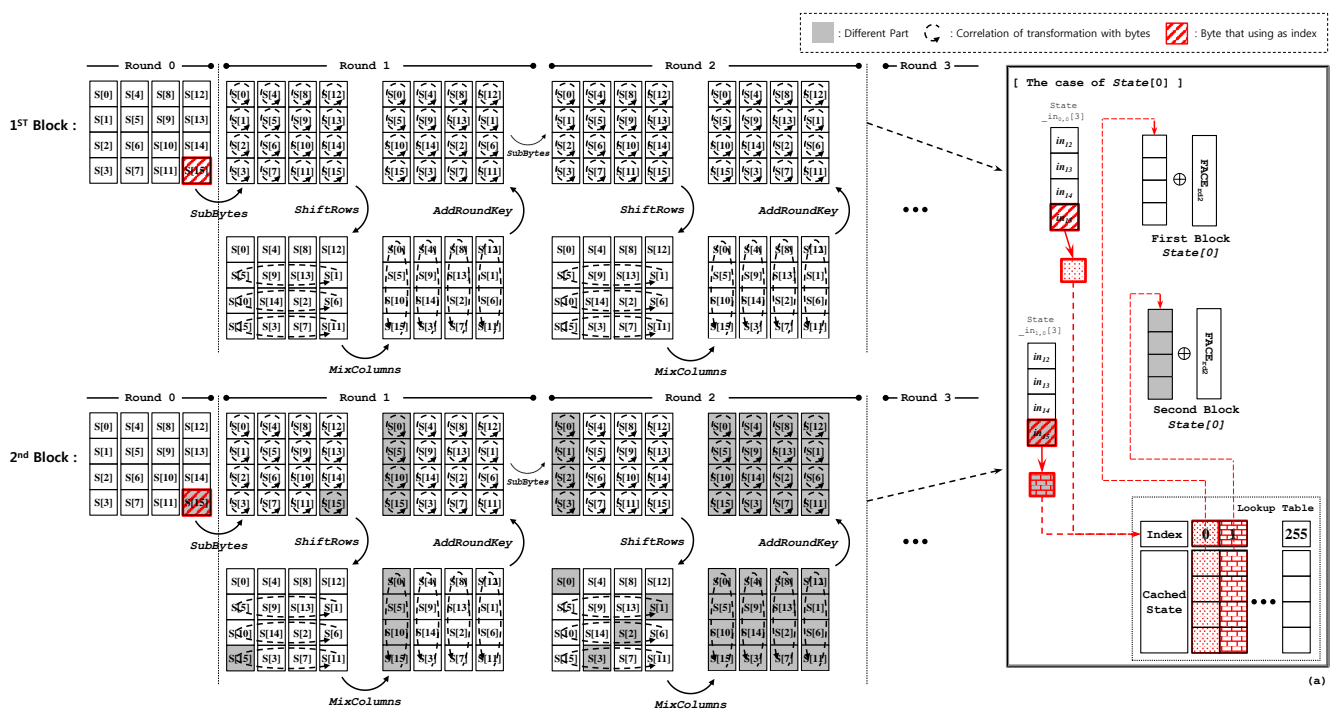

Figure 7: $\mathrm{FACE}_{r d 2+}$ : The process of AES round 1, round 2, and $\mathrm{FACE}_{r d 2+} \cdot$ (a) shows how to refer the pre-computation values and how to calculate the result of round 2 .

$$
\begin{aligned}
\left(\begin{array}{c}
S^{\prime}[0] \\
S^{\prime}[5] \\
S^{\prime}[10] \\
S^{\prime}[15]
\end{array}\right)= & \left(\begin{array}{llll}
2 & 3 & 1 & 1 \\
1 & 2 & 3 & 1 \\
1 & 1 & 2 & 3 \\
3 & 1 & 1 & 2
\end{array}\right) \otimes\left(\begin{array}{c}
S[0] \\
S[5] \\
S[10] \\
S[15]
\end{array}\right) \oplus\left(\begin{array}{l}
r k_{2,0} \\
r k_{2,1} \\
r k_{2,2} \\
r k_{2,3}
\end{array}\right) \\
= & \left(\begin{array}{l}
2 \cdot S[0] \oplus 3 \cdot S[5] \oplus 1 \cdot S[10] \oplus 1 \cdot S[15] \oplus r k_{2,0} \\
1 \cdot S[0] \oplus 2 \cdot S[5] \oplus 3 \cdot S[10] \oplus 1 \cdot S[15] \oplus r k_{2,1} \\
1 \cdot S[0] \oplus 1 \cdot S[5] \oplus 2 \cdot S[10] \oplus 3 \cdot S[15] \oplus r k_{2,2} \\
3 \cdot S[0] \oplus 1 \cdot S[5] \oplus 1 \cdot S[10] \oplus 2 \cdot S[15] \oplus r k_{2,3}
\end{array}\right)
\end{aligned}
$$

Except for the intermediate result that is cached by $F A C E_{r d 2}$, the remaining data are $2 \cdot S[0]$ for $S^{\prime}[0], 1 \cdot S[0]$ for $S^{\prime}[5], 1 \cdot S[0]$ for $S^{\prime}[10]$, and $3 \cdot S[0]$ for $S^{\prime}[15]$. All of this remaining data are related to $\mathrm{S}[0]$. Similar situations can be seen in other rout ${ }_{i, 2}[i](1 \leq i \leq 3)$. Similarly, the remaining data of rout $_{i, 2}[1], \operatorname{rout}_{i, 2}[2]$, and rout $_{i, 2}[3]$ are related to $S[3]$, $S[2]$, and $S[1]$ respectively. Recall that the SubBytes() and ShiftRows () transformations do not affect other bytes. Thus, these four bytes $(S[0], S[1], S[2], S[3])$ are considered to be the same as $\operatorname{rin}_{i, 2}[0]$. This means that the remaining intermediate data is determined by $\operatorname{rin}_{i, 2}[0]$.

According to $\mathrm{FACE}_{r d 1+}$, rout ${ }_{i, 1}[0]\left(\operatorname{rin}_{i, 2}[0]\right)$ is determined by $i n_{15}$. And the remaining intermediate data for $\mathrm{FACE}_{r d 2+}$ is determined by $\operatorname{rin}_{i, 2}[0]$. In the end, we can generate a temporary look-up table and use $i n_{15}$ as an index. If we create a pre-computation table for $\mathrm{FACE}_{r d 2+}$, we need an additional $4 \mathrm{~KB}(16 \times 256)$ of memory because the remaining intermediate data is 4 bytes for each rout r,2 $_{2}[i](0 \leq i \leq 3)$, and $i n_{15}$ changes from $0 \times 00$ to $0 x F F$. FACE $E_{r d 2+}$ can reuse these cached data while processing $2^{40}$ consecutive blocks. The frequency of updates is equal to that of $\mathrm{FACE}_{r d 1+}$.

\section{Evaluations}

\subsection{Implementation}

The proposed method (FACE) can be employed in any AES CTR implementation, regardless of the platform, environment, or implementation method. We implement FACE by 
Table 2: Environments used for evaluation

\begin{tabular}{|c|c|c|c|}
\hline & Test Environment 1 & Test Environment 2 & Test Environment 3 \\
\hline \hline $\mathrm{CPU}$ & Intel Core 2 Quad Q9550 & Intel Core i7 4770K & Intel Core i7 8700K \\
\hline $\begin{array}{c}\mathrm{CPU} \\
\text { Frequency }\end{array}$ & $2.8 \mathrm{GHz}$ & $3.5 \mathrm{GHz}$ & $3.7 \mathrm{GHz}$ \\
\hline RAM & $4 \mathrm{~GB}$ & $8 \mathrm{~GB}$ & $16 \mathrm{~GB}$ \\
\hline OS & Linux 3.19.0-32 & Linux 3.19.0-32 & Linux 4.13.0-36 \\
& $\mathrm{x} 86 \_64$ & x86_64 64 & 64 \\
\hline
\end{tabular}

modifying the AES source code contained in the open-source libraries (OpenSSL [Pro] and Crypto $++[\mathrm{Lib}])$. OpenSSL provides AES source code for both the table-based and the bitsliced cases. In particular, bitsliced AES is implemented based on [KS09], which is known as the fastest bitsliced AES CTR software implementation with one core. We analyzed the source code of [KS09] and compared it with the bitsliced implementation of OpenSSL. As a result, we found that the two codes are very similar and almost identical (as OpenSSL commented on its source code). Moreover, OpenSSL left a record of its own comparison with [KS09], and OpenSSL shows better performance. We think OpenSSL is more practical because it supports 192- and 256-bit keys, unlike [KS09]. Thus, we selected OpenSSL as our comparison target of bitsliced implementation. The AES implementation of Crypto++ supports AES-NI instructions via compiler intrinsics. It provides two encryption interfaces; one is for processing one 128-bit block per call (which we denoted as " $1 \mathrm{x} 1$ "), and the other is for processing four 128-bit blocks per call (which we denoted as " $4 \mathrm{x} 1$ "). To the best of our knowledge (based on eSTREAM/Crypto++ benchmark and other literature), AES-NI-based implementation of Crypto++ can be considered the fastest one. Thus, we selected Crypto++ as our comparison target of AES-NI-based implementation. For the experiments on AES-NI-based FACE, we prepared two kinds of implementations: $\mathrm{R} 1$ and R2. R1 is an implementation, which leverages FACE up to round 1. R2 is an implementation, which adopts FACE up to round 2.

For a fair comparison, we did not re-code the existing strategy into our own implementation. Since the same strategy can record different performance depending on the quality of code, and consequently, it can be misunderstood that the reason of improvements comes from the quality of the code we produced. Thus, we opted to adopt an existing implementation and use it as our own. And then we made only minor modifications to the existing code in order to apply our strategy. Except for our new strategy, all other conditions remain the same. This can be confirmed by comparing our target open-source with the appendix.

The five techniques of FACE can be chosen selectively, with consideration for the environment in which FACE will be applied. When code size or cache space is an issue, our techniques can be applied by choosing the most efficient combinations.

As a result of applying FACE to existing implementations, table-based FACE needs 12 instructions up to round 2, whereas the existing implementation [Pro] needs 128 instructions. Considering the cache update process, which occurs once for every 256 blocks, table-based FACE needs 12.4 instructions on average. Bitsliced FACE requires 74 instructions up to round 2, whereas [KS09] requires 618 instructions. As with the table-based FACE, considering the cache update procedure, bitsliced FACE needs 91.9 instructions on average. AES-NI-based FACE requires 1 intrinsic instruction, 1 memory reference, and 1 arithmetic instruction (simply, 3 instructions) up to round 2 , whereas [Lib] requires 3 intrinsic instructions and 3 memory references (simply, 6 instructions). According to the Intel instruction latency and throughput [Cor18], a briefly calculated throughput (required cycle) for AES-NI-based FACE is 0.83 up to round 2, whereas for [Lib] it is 3.08. Considering the cache update procedure as it is in other methods, AES-NI-based FACE requires (simply) 3.02 instructions on average. 
Table 3: Performance comparison of AES-CTR implementations in cycles/byte

\begin{tabular}{|c|c|c|c|c|c|c|c|c|c|c|c|c|c|c|c|}
\hline \multirow{3}{*}{ Platform } & \multirow{3}{*}{\multicolumn{2}{|c|}{$\begin{array}{c}\text { Implementation } \\
\text { Method }\end{array}$}} & \multirow{3}{*}{ Target } & \multicolumn{12}{|c|}{ Input (Plaintext) Size } \\
\hline & & & & \multicolumn{3}{|c|}{1024 bytes } & \multicolumn{3}{|c|}{4096 bytes } & \multicolumn{3}{|c|}{20480 bytes } & \multicolumn{3}{|c|}{40960 bytes } \\
\hline & & & & 128 & 192 & 256 & 128 & 192 & 256 & 128 & 192 & 256 & 128 & 192 & 256 \\
\hline \multirow{4}{*}{ Test Env 1} & \multirow{2}{*}{\multicolumn{2}{|c|}{ Table-based }} & OpenSSL & 15.849 & 18.302 & 20.710 & 15.786 & 18.272 & 20.680 & 15.766 & 18.249 & 20.665 & 15.768 & 18.238 & 20.659 \\
\hline & & & This Paper & 12.452 & 14.947 & 17.336 & 12.407 & 14.936 & 17.329 & 12.394 & 14.911 & 17.321 & 12.399 & 14.913 & 17.326 \\
\hline & \multirow{2}{*}{\multicolumn{2}{|c|}{ Bitsliced }} & [KS09] & 8.014 & 9.495 & 10.960 & $7.811(7.59)$ & 9.251 & 10.686 & 7.763 & 9.195 & 10.624 & 7.764 & 9.192 & 10.618 \\
\hline & & & This Paper & 6.754 & 8.180 & 9.607 & $6.408(6.347)$ & 7.797 & 9.180 & 6.364 & 7.755 & 9.119 & 6.360 & 7.752 & 9.108 \\
\hline \multirow{10}{*}{ Test Env 2} & \multirow{2}{*}{\multicolumn{2}{|c|}{ Table-based }} & OpenSSL & 10.562 & 12.309 & 14.036 & 10.553 & 12.348 & 14.067 & 10.529 & 12.276 & 14.023 & 10.528 & 12.276 & 14.023 \\
\hline & & & This Paper & 8.380 & 10.085 & 11.808 & 8.344 & 10.064 & 11.797 & 8.371 & 10.067 & 11.810 & 8.368 & 10.071 & 11.808 \\
\hline & \multirow{2}{*}{\multicolumn{2}{|c|}{ Bitsliced }} & [KS09] & 5.687 & 6.745 & 7.803 & 5.530 & 6.554 & 7.573 & 5.514 & 6.491 & 7.511 & 5.500 & 6.482 & 7.495 \\
\hline & & & This Paper & 4.696 & 5.737 & 6.787 & 4.429 & 5.455 & 6.476 & 4.398 & 5.407 & 6.425 & 4.397 & 5.406 & 6.422 \\
\hline & \multirow{6}{*}{ AES-NI } & \multirow{3}{*}{$1 \times 1$} & Crypto++ & 2.540 & 2.957 & 3.321 & 2.506 & 2.896 & 3.283 & 2.698 & 3.083 & 3.482 & 2.695 & 3.080 & 3.477 \\
\hline & & & This Paper (R1) & 1.025 & 1.267 & 1.556 & 1.018 & 1.253 & 1.552 & 1.073 & 1.301 & 1.578 & 1.071 & 1.294 & 1.558 \\
\hline & & & This Paper (R2) & 0.927 & 1.160 & 1.383 & 0.917 & 1.146 & 1.377 & 1.040 & 1.188 & 1.398 & 1.040 & 1.189 & 1.398 \\
\hline & & \multirow{3}{*}{$4 \times 1$} & Crypto++ & 0.730 & 0.861 & 0.984 & 0.704 & 0.840 & 0.983 & 0.688 & 0.824 & 0.969 & 0.684 & 0.822 & 0.967 \\
\hline & & & This Paper (R1) & 0.634 & 0.781 & 0.923 & 0.623 & 0.769 & 0.920 & 0.621 & 0.765 & 0.911 & 0.620 & 0.765 & 0.910 \\
\hline & & & This Paper (R2) & 0.592 & 0.727 & \begin{tabular}{|l|}
0.869 \\
\end{tabular} & 0.580 & 0.714 & 0.858 & 0.578 & 0.711 & 0.857 & 0.578 & 0.711 & 0.857 \\
\hline \multirow{10}{*}{ Test Env 3} & \multirow{2}{*}{\multicolumn{2}{|c|}{ Table-based }} & OpenSSL & 9.374 & 10.948 & 12.645 & 9.223 & 10.788 & 12.496 & 9.083 & 10.354 & 11.822 & 8.716 & 10.087 & 11.644 \\
\hline & & & This Paper & 7.185 & 8.741 & 10.346 & 7.114 & 8.726 & 10.230 & 7.081 & 8.408 & 9.847 & 6.855 & 8.203 & 9.647 \\
\hline & \multirow{2}{*}{\multicolumn{2}{|c|}{ Bitsliced }} & [KS09] & 5.273 & 6.108 & 7.254 & 5.172 & 6.074 & 7.079 & 5.097 & 5.999 & 6.995 & 5.032 & 5.879 & 6.952 \\
\hline & & & This Paper & 4.339 & 5.356 & 6.278 & 3.932 & 4.984 & 5.987 & 4.006 & 4.945 & 5.873 & 3.812 & 4.691 & 5.571 \\
\hline & \multirow{6}{*}{ AES-NI } & \multirow{3}{*}{$1 \times 1$} & Crypto++ & 1.665 & 1.871 & 2.059 & 1.625 & 1.847 & 2.043 & 1.617 & 1.832 & 2.029 & 1.611 & 1.807 & 2.021 \\
\hline & & & This Paper (R1) & 0.778 & 0.867 & 0.986 & 0.739 & 0.827 & \begin{tabular}{|l|}
0.959 \\
\end{tabular} & 0.737 & 0.822 & 0.956 & 0.726 & 0.819 & 0.948 \\
\hline & & & This Paper (R2) & 0.703 & 0.786 & 0.880 & 0.662 & 0.775 & 0.867 & 0.659 & 0.732 & 0.874 & 0.658 & 0.733 & 0.876 \\
\hline & & \multirow{3}{*}{$4 \times 1$} & Crypto++ & 0.551 & 0.669 & 0.767 & 0.547 & 0.642 & 0.758 & 0.537 & 0.636 & 0.745 & 0.531 & 0.622 & 0.739 \\
\hline & & & This Paper (R1) & 0.513 & 0.607 & 0.706 & 0.494 & 0.586 & 0.698 & 0.483 & 0.581 & 0.684 & 0.473 & 0.573 & 0.677 \\
\hline & & & This Paper (R2) & 0.450 & 0.547 & 0.638 & 0.441 & 0.533 & 0.636 & 0.442 & 0.539 & 0.624 & 0.434 & 0.539 & 0.625 \\
\hline
\end{tabular}

We note that the code used in the experiments is identical, regardless of the test environment. For example, we do not use additional optimization techniques for the bitsliced FACE in the test environment 2 and 3 (microarchitectures that provide a 256-bit AVX instruction set). This is guaranteed because the bitsliced method is implemented using low-level programming language (Assembly). In conclusion, when implementing FACE, we do not take advantage of other optimizations that leverage special features of the test environment.

\subsection{Experimental Results}

To evaluate our method, FACE, we first verify the proposed technique and its implementation using test vectors with different input lengths and key lengths. The results show that the table-based, bitsliced, and AES-NI-based FACE all work correctly. Then, we measure the throughput of FACE that was employed in table-based, bitsliced, and AES-NI-based implementations on Intel Core 2 Quad and Core i7 processors. Then, we compare our results to those of a default implementation contained in the open-source libraries. A description of the environments used for the evaluation is given in Table 2. All tests were conducted using only one core.

A comprehensive comparison of the evaluation results is summarized in Table 3 . We measure the throughput using three key lengths (128, 192, and 256 bits), while changing the size of the input blocks to 1024, 4096, 20480, and 40960 bytes. We also measure the performance of the base code that FACE leveraged, within the same environments. Since all the experimental environments can not be the same, all experimental results, except those recorded in the literature using the same environment, are presented with our own measuring results in our environments.

In the case of the table-based implementation, our implementation of AES CTR 
achieves a performance improvement of $15 \%-20 \%$. This result shows that table-based FACE achieves similar performance to AES CTR, which has lower-level security (e.g., the performance of table-based FACE-192 $\approx$ the performance of the default AES-128). We compared our results with table-based implementation of OpenSSL. However, there exists an outperformed result, which records 10.57 cycles/byte on Intel Core 2 Quad Q9550 [BS08]. We did not try to apply our strategy to [BS08]. Because our main targets are bitsliced and AES-NI-based implementations. Additionally, we believe that the table-based implementation is still not of much interest; it has reached its performance limits, and also has a security concern. We intended to simply show, using the experiments, that our proposal can be applied to table-based. It was not our goal to achieve state-of-the-art results with the table-based implementation.

Bitsliced FACE needs 6.41 cycles/byte for an input size of 4096 bytes and a 128 bit key length, where the previous work recorded 7.81 cycles/byte in test environment 1 . It appears that the performance of [KS09] $(7.81 \mathrm{cpb})$ is worse than the reported throughput in [KS09] $(7.59 \mathrm{cpb})$. Our insight into this is that the results of our experiments include key transformation (a conversion of round keys from a table-based representation to bitslice form) at the beginning of the encryption phase (the result of [KS09] did not include such key transformation cost in the encryption phase). Also, for a common usage, [KS09] implementation of OpenSSL adds more routines to support other key lengths (192 and 256 bits) and employs different byte order. If we exclude the key transformation process, [KS09] implementation of OpenSSL requires 7.75 cycles/byte according to our experiments. Bitsliced FACE without key transformation records 6.35 cycles/byte). Further, bitsliced FACE needs 3.93 cycles/byte, while [KS09] implementation of OpenSSL records 5.17 cycles/byte on a recent Intel Core i7 (Test Environment 3). Our bitsliced implementation of AES CTR is about $20 \%$ faster than those in previous works, and the result of 6.41 cycles/byte for an input size of 4096 bytes is the highest throughput ever achieved in a PC environment (without AVX or AES-NI). Thanks to the characteristic of FACE that uses repetitive data, the throughput of FACE increases as the length of the input block increases. Finally, bitsliced FACE records 6.36 cycles/byte for an input size of 40960 bytes with a 128-bit key.

Our AES-NI-based FACE needs 0.44 cycles/byte for an input size of 4096 bytes and a 128 bit key length, where AES-NI-based AES CTR implementation of [Lib] recorded 0.54 cycles/byte on Intel Core i7 8700K. Further, AES-NI-based FACE records 0.43 cycles/byte for an input size of 40960 bytes with a 128-bit key, whereas [Lib] needs 0.53 cycles/byte. The result of 0.44 cycles/byte is also the highest throughput ever achieved in an AES CTR implementation with AES-NI.

The speedups shown in our experiments are on the same level as (or even exceed) the simple theoretical model of simply skipping the first two rounds. FACE targets the first two rounds of AES. AES-128 has 10 rounds and AES-256 has 14 rounds. If the first two rounds could be skipped completely, the expectable speedups are up to $20 \%$ and $14 \%$, respectively. Our insight into the reason of speedups, which are shown to be as good as this kind of simplistic model, can be described briefly as follows. First, FACE can be considered as completely skipping the first two rounds like the simple theoretical model. After only 1 XOR operation, the first two rounds are completed, and this cost is equal to initial whitening. Second, FACE additionally skips several operations; copying input to local variable, increasing counter value (if the increment is pre-defined, there is no need to increase counter for every block), and in case of bitslice method, a costly transformation of input to bitslice form as well as ShiftRows of round 3. Such operations are also included in the cached value of FACE. Third, FACE has benefits (only for table-based) from the optimization of compiler due to its simplified high-level PL code, and its assumed benefit is about $2-3 \%$. Lastly, the improvement of "AES-NI ( $1 \times 1)$ " is a special case, because the cost of memory copies and function calls are considerably reduced by FACE compared 
Table 4: Comparison of key-scheduling performances

\begin{tabular}{|c|c|c|c|c|c|c|}
\hline \multirow{2}{*}{ Platform } & \multirow{2}{*}{$\begin{array}{c}\text { Implementation } \\
\text { Method }\end{array}$} & \multirow{2}{*}{ Measurement } & \multirow{2}{*}{ Target } & \multicolumn{3}{|c|}{ Key Size } \\
\hline & & & & AES-128 & AES-192 & AES-256 \\
\hline \multirow{12}{*}{ Test Env 1} & \multirow{6}{*}{ Table-based } & \multirow{2}{*}{$\begin{array}{c}\text { Cycles } \\
\text { for key setup }\end{array}$} & OpenSSL & 1776 & 197 & 259 \\
\hline & & & This Paper & 6067 & 6134 & 6276 \\
\hline & & \multirow{2}{*}{$\begin{array}{c}\text { Times } \\
\text { for key setup }\end{array}$} & OpenSSL & $0.062 \mu \mathrm{s}$ & $0.070 \mu \mathrm{s}$ & $0.091 \mu \mathrm{s}$ \\
\hline & & & This Paper & $2.147 \mu \mathrm{s}$ & $2.170 \mu \mathrm{s}$ & $2.221 \mu \mathrm{s}$ \\
\hline & & \multicolumn{2}{|c|}{ Cycle Overhead } & 5891 & 5937 & 6017 \\
\hline & & \multicolumn{2}{|c|}{ Time Overhead } & $2.085 \mu \mathrm{s}$ & $2.100 \mu \mathrm{s}$ & $2.130 \mu \mathrm{s}$ \\
\hline & \multirow{6}{*}{ Bitsliced } & \multirow{2}{*}{$\begin{array}{c}\text { Cycles } \\
\text { for key setup }\end{array}$} & OpenSSL & 383 & 389 & 478 \\
\hline & & & This Paper & 8901 & 8908 & 8992 \\
\hline & & \multirow{2}{*}{$\begin{array}{c}\text { Times } \\
\text { for key setup }\end{array}$} & OpenSSL & $0.074 \mu \mathrm{s}$ & $0.075 \mu \mathrm{s}$ & $0.083 \mu \mathrm{s}$ \\
\hline & & & This Paper & $3.146 \mu \mathrm{s}$ & $3.152 \mu \mathrm{s}$ & $3.177 \mu \mathrm{s}$ \\
\hline & & \multicolumn{2}{|c|}{ Cycle Overhead } & 8518 & 8519 & 8514 \\
\hline & & \multicolumn{2}{|c|}{ Time Overhead } & $3.072 \mu \mathrm{s}$ & $3.077 \mu \mathrm{s}$ & $3.094 \mu \mathrm{s}$ \\
\hline \multirow{22}{*}{ Test Env 3} & \multirow{6}{*}{ Table-based } & \multirow{2}{*}{$\begin{array}{c}\text { Cycles } \\
\text { for key setup }\end{array}$} & OpenSSL & 121 & 137 & 172 \\
\hline & & & This Paper & 3643 & 3662 & 3724 \\
\hline & & \multirow{2}{*}{$\begin{array}{c}\text { Times } \\
\text { for key setup }\end{array}$} & OpenSSL & $0.033 \mu \mathrm{s}$ & $0.036 \mu \mathrm{s}$ & $0.050 \mu \mathrm{s}$ \\
\hline & & & This Paper & $0.980 \mu \mathrm{s}$ & $0.993 \mu \mathrm{s}$ & $1.010 \mu \mathrm{s}$ \\
\hline & & \multicolumn{2}{|c|}{ Cycle Overhead } & 3522 & 3525 & 3552 \\
\hline & & & $0.947 \mu \mathrm{s}$ & $0.957 \mu \mathrm{s}$ & $0.960 \mu \mathrm{s}$ \\
\hline & \multirow{6}{*}{ Bitsliced } & \multirow{2}{*}{$\begin{array}{c}\text { Time Ove } \\
\text { Cycles } \\
\text { for key setup }\end{array}$} & OpenSSL & 242 & 254 & 306 \\
\hline & & & This Paper & 5778 & 5783 & 5802 \\
\hline & & \multirow{2}{*}{$\begin{array}{c}\text { Times } \\
\text { for key setup }\end{array}$} & OpenSSL & $0.066 \mu \mathrm{s}$ & $0.069 \mu \mathrm{s}$ & $0.086 \mu \mathrm{s}$ \\
\hline & & & This Paper & $1.577 \mu \mathrm{s}$ & $1.591 \mu \mathrm{s}$ & $1.601 \mu \mathrm{s}$ \\
\hline & & \multicolumn{2}{|c|}{ Cycle Overhead } & 5536 & 5529 & 5496 \\
\hline & & \multicolumn{2}{|c|}{ Time Overhead } & $1.511 \mu \mathrm{s}$ & $1.522 \mu \mathrm{s}$ & $1.515 \mu \mathrm{s}$ \\
\hline & \multirow{10}{*}{ AES-NI } & & Crypto ++ & 145 & 173 & 212 \\
\hline & & Cycles for key setup & This Paper (R1) & 680 & 749 & 783 \\
\hline & & & This Paper (R2) & 7568 & 7628 & 7618 \\
\hline & & & Crypto++ & $0.039 \mu \mathrm{s}$ & $0.047 \mu \mathrm{s}$ & $0.058 \mu \mathrm{s}$ \\
\hline & & Times for key setup & This Paper (R1) & $0.184 \mu \mathrm{s}$ & $0.196 \mu \mathrm{s}$ & $0.205 \mu \mathrm{s}$ \\
\hline & & & This Paper (R2) & $1.994 \mu \mathrm{s}$ & $2.067 \mu \mathrm{s}$ & $2.054 \mu \mathrm{s}$ \\
\hline & & Cycle Overh & $\operatorname{ad}(\mathrm{R} 1)$ & 535 & 576 & 571 \\
\hline & & Cycle Overh & ad (R2) & 7428 & 7455 & 7406 \\
\hline & & Time Overh & $\operatorname{ad}(\mathrm{R} 1)$ & $0.145 \mu \mathrm{s}$ & $0.149 \mu \mathrm{s}$ & $0.147 \mu \mathrm{s}$ \\
\hline & & Time Overh & ad (R2) & $1.955 \mu \mathrm{s}$ & $2.020 \mu \mathrm{s}$ & $1.996 \mu \mathrm{s}$ \\
\hline
\end{tabular}

to $4 \times 1$. It demonstrates that such operations can be a significant burden.

It seems that the performance improvement is modest, but in effect, such an improvement leads to a difference of hundreds of megabytes per second in throughput. Our experimental results demonstrate that FACE can be applied to any AES CTR implementation, regardless of the implementation method, and can improve performance in various environments.

When applying the technique discussed in section 4.3 or 4.5 , our proposed method can create pre-computation values in the initialization stage. This may be an additional burden in terms of efficiency. Therefore, we measure the processing time and cycle counts for the initialization to show that the generation of pre-computation values does not require significant overhead. Table 4 shows the evaluation results of the initialization stage (key scheduling). The key-schedule process of the bitsliced method includes a transformation that converts round keys from a table-based representation to a bitslice representation. All of AES-128, AES-192, and AES-256 require little overhead to generate the temporary lookup table. Thus, the generation of pre-computation values leads to marginal overhead with respect to the performance of FACE.

\section{Discussion}

As cache-timing attacks have become a promising attacks on software-based AES implementations, they have become significant security threats for AES implementations. Cache-timing attacks on AES exploit the timing variability of data loads from memory while implementations make heavy use of lookup tables. It seems that our proposed 
methods are vulnerable to timing attacks because of the use of pre-computed lookup tables. We note that the vulnerabilities caused by timing attacks on FACE are dependent on the adopting implementation method because FACE can be employed in any AES CTR implementation, regardless of implementation method (i.e. table-based, bitsliced, and AES-NI-based). If FACE is applied to the table-based method, the result is also vulnerable to timing attacks. On the other hand, if FACE is applied to the bitsliced method, which offers timing-attack resistance, the result is also cache-timing-attack resistant. It means that the vulnerabilities caused by timing attacks on an AES implementation are not affected by our proposed methods. There are several reasons to explain this.

Several timing attacks against AES make it possible to recover secret information. This is because the indices of the table in the existing table-based implementation are related to secret information. In contrast, FACE adds temporary tables for intermediate values and the indices of our generated tables are independent of secret information. In FACE $r d 0$, $\mathrm{FACE}_{r d 1}$, and $\mathrm{FACE}_{r d 2}$, the size of the generated table is extremely small and the indices of the tables are fixed. The size of tables are 12 bytes for each $\mathrm{FACE}_{r d 0}$ and $\mathrm{FACE}_{r d 1}$, and 16 bytes for $\mathrm{FACE}_{r d 2}$. Modern cache stores groups of bytes in blocks of fixed sizes, called cache lines. Common cache line sizes are 32 bytes for a Pentium III, and 64 and 128 bytes for more recent processors. Data is transferred between main memory and cache in blocks of cache line size. Therefore, all of our generated values in the tables for each phase $\left(\mathrm{FACE}_{r d 0}, \mathrm{FACE}_{r d 1}\right.$, and $\left.\mathrm{FACE}_{r d 2}\right)$ always share a line in the cache on any cache line size (cache hit). However, the timing variations, which cause information leakage, may exist even if loading occurs only within a fixed cache line. Osvik et al. [OST06] and Bernstein [Ber05] warned that even if secret-dependent accesses are at a finer than cache line granularity, access to different offsets within cache lines may leak information due to cache-bank conflicts. Finally, Yarom et al. [YGH17] demonstrated that the first side-channel attack, which exploits cache-bank conflicts, is feasible on the Sandy Bridge microarchitecture. Such an attack is viable when a secret-dependent access to cache banks exists. But in FACE, the indices of the table lookups are always constant as 0 to 2 in the phase of $\mathrm{FACE}_{r d 0}$ and $\mathrm{FACE}_{r d 1}$, and 0 to 3 in the phase of $\mathrm{FACE}_{r d 2}$ (all content of tables are used in round operation). In this case, there is no secret-dependent access patterns when FACE accesses the cache. Currently, cache-bank conflicts are no longer an issue since the Haswell processors [Cor18].

In the phase of $\mathrm{FACE}_{r d 1+}$ or $\mathrm{FACE}_{r d 2+}$, our method generates a larger table (1024 bytes for $\mathrm{FACE}_{r d 1+}, 4096$ bytes for $\left.\mathrm{FACE}_{r d 2+}\right)$. This table might cause a cache miss. However, the index of the table is merely a part of a counter value that does not need to be secret (as generally known, keeping the counter value secret adds no security). More precisely, the lookup index is the last byte of the counter, and it even increases linearly. There is no secret-dependent access information while loading data from the table. Furthermore, there is no operation that includes the loaded table value and the secret. In addition, the index is increased by one if the counter is increased by one. Thus, on $\mathrm{FACE}_{r d 1+}$ phase, cache miss caused periodically for every 16 consecutive blocks when only one (64 bytes) cache line is used (caused for every 4 consecutive blocks on $F A C E_{r d 2}+$ phase). The timing variability of data loads from memory due to cache miss would present cyclically, and there is no secret-dependent timing variability in the data loads from the table while processing consecutive blocks for each cache miss.

These features show that our approach does not cause additional vulnerabilities to known timing attacks.

\section{Conclusion}

The AES CTR mode is used in encryption/decryption operations such as streaming services that require high-speed processing because it enables parallel processing. Efforts to improve 
the efficiency of AES CTR mode implementation in software have been realized using the bitsliced method. Although performance improvements in algorithms for specialized platforms lack scalability, improvements in the implementation logic of AES can be applied without depending on operating architectures and implementation methods.

This paper proposed FACE, which can improve the performance of the AES CTR mode by using repetitive data. FACE reduces the number of unnecessary calculations during the operation in order to preserve computational resources. To accomplish this, we leverage that very small changes occur in successive blocks in the AES CTR mode, which can be cached and reused. FACE can be employed in any AES CTR implementation, regardless of implementation method (i.e. table-based, bitsliced, and AES-NI-based). As a result, our experimental results are approximately $15 \%-20 \%$ more efficient than those of previously reported methods using a single core. In addition, none of the techniques introduced in this paper are platform specific. Thus, when we apply this technique to implementations of the AES CTR mode, we can expect to achieve performance improvements regardless of the platform or environment. Therefore, FACE provides a computational advantage over high-profile I/O and network services, such as Gigabit ethernet, the Wireless Gigabit Alliance (WiGig) 1.1 [All10] network, and USB 3.0.

We can consider applying our strategy to CAESAR finalist Deoxys [JNPS16], since Deoxys also uses a counter in the tweak input while the plaintext remains unchanged. The caching strategy for Deoxys is slightly more complex than AES because the differences between successive blocks are caused not only by the round transformation but also by the tweakey schedule algorithm. Nonetheless, the additional difference is not considerable. For example, at the end of the first round, the difference is increased only one byte compared to FACE, and there also exists the characteristic of being repeated. It would be interesting to verify whether our caching strategy can be applied to other algorithms that have similar characteristics to the AES CTR mode. This is the subject of our future work to examine the extendability of our caching strategies.

\section{Acknowledgements}

We would like to thank the anonymous reviewers for their valuable and helpful comments in improving the quality of this work. This work was supported by Institute for Information \& communications Technology Promotion(IITP) grant funded by the Korea government(MSIT) (No. R7117-16-0161, Anomaly Detection Framework for Autonomous Vehicles).

\section{References}

[All08] The Open Mobile Alliance. DRM Content Format Approved Version 2.1.2, 2008.

[All10] WiGig Alliance. Defining the future of multi-gigabit wireless communications. WiGig White Paper, 2010.

$\left[\mathrm{BBF}^{+}\right.$02] Guido Bertoni, Luca Breveglieri, Pasqualina Fragneto, Marco Macchetti, and Stefano Marchesin. Efficient software implementation of aes on 32-bit platforms. In International Workshop on Cryptographic Hardware and Embedded Systems, pages 159-171. Springer, 2002.

[Ber05] Daniel J Bernstein. Cache-timing attacks on aes. 2005.

[Bih97] Eli Biham. A fast new des implementation in software. In International Workshop on Fast Software Encryption, pages 260-272. Springer, 1997. 
$\left[\mathrm{BMN}^{+}\right.$04] Mark Baugher, D McGrew, M Naslund, E Carrara, and Karl Norrman. The secure real-time transport protocol (SRTP). RFC 3711, 2004.

[BS08] Daniel J Bernstein and Peter Schwabe. New aes software speed records. In International Conference on Cryptology in India, pages 322-336. Springer, 2008.

[Cor18] Intel Corporation. Intel 64 and IA-32 architectures optimization reference manual. Order Number: 248966-040, April 2018.

[CYW03] Francois Charot, Eslam Yahya, and Charles Wagner. Efficient modularpipelined aes implementation in counter mode on altera fpga. In International Conference on Field Programmable Logic and Applications, pages 282-291. Springer, 2003.

[DR13] Joan Daemen and Vincent Rijmen. The design of Rijndael: AES-the advanced encryption standard. Springer Science \& Business Media, 2013.

[GCVRSPGP10] José M Granado-Criado, Miguel A Vega-Rodríguez, Juan M SánchezPérez, and Juan A Gómez-Pulido. A new methodology to implement the aes algorithm using partial and dynamic reconfiguration. INTEGRATION, the VLSI journal, 43(1):72-80, 2010.

[Gue10] Shay Gueron. Intel@ advanced encryption standard (AES) new instructions set. Intel Corporation, 2010.

[JNPS16] Jérémy Jean, Ivica Nikolic, Thomas Peyrin, and Yannick Seurin. Deoxys v1.41. CAESAR Finalists, October 2016. https://competitions.cr. yp.to/caesar-submissions.html.

[KS09] Emilia Käsper and Peter Schwabe. Faster and timing-attack resistant aes-gcm. In Cryptographic Hardware and Embedded Systems-CHES 2009, pages 1-17. Springer, 2009.

[LB13] Bin Liu and Bevan M Baas. Parallel aes encryption engines for many-core processor arrays. IEEE transactions on computers, 62(3):536-547, 2013.

[Lib] CRYPTO++ Library. http://www.cryptopp.com.

[Mat06] Mitsuru Matsui. How far can we go on the x64 processors? In International Workshop on Fast Software Encryption, pages 341-358. Springer, 2006.

[McG02] David McGrew. Counter mode security: Analysis and recommendations. Cisco Systems, November, 2:4, 2002.

[MF05] Mitsuru Matsui and Sayaka Fukuda. How to maximize software performance of symmetric primitives on pentium iii and 4 processors. In International Workshop on Fast Software Encryption, pages 398-412. Springer, 2005.

[MN07] Mitsuru Matsui and Junko Nakajima. On the power of bitslice implementation on intel core 2 processor. In International Workshop on Cryptographic Hardware and Embedded Systems, pages 121-134. Springer, 2007.

[MS04] Sumio Morioka and Akashi Satoh. A 10-gbps full-aes crypto design with a twisted bdd s-box architecture. IEEE Transactions on Very Large Scale Integration (VLSI) Systems, 12(7):686-691, 2004. 
[NIS01a] NIST. Advanced Encryption Standard (AES), 2001. http://csrc.nist. gov/publications/fips/fips197/fips-197.pdf.

[NIS01b] NIST. Recommendation for Block Cipher Modes of Operation (Methods and Techniques), 2001. http://csrc.nist.gov/publications/ nistpubs/800-38a/sp800-38a.pdf.

[NIS04] NIST. Recommendation for Block Cipher Modes of Operation:The CCM Mode for Authentication and Confidentiality, 2004. http://csrc.nist.gov/publications/nistpubs/800-38C/ SP800-38C_updated-July20_2007.pdf.

[NIS07] NIST. Recommendation for Block Cipher Modes of Operation:Galois/Counter Mode (GCM) and GMAC, 2007. http://csrc. nist.gov/publications/nistpubs/800-38D/SP-800-38D .pdf.

[OST06] Dag Arne Osvik, Adi Shamir, and Eran Tromer. Cache attacks and countermeasures: the case of aes. In Cryptographers' Track at the RSA Conference, pages 1-20. Springer, 2006.

[Pro] The OpenSSL Project. http://www.openssl.org.

$\left[\mathrm{QSH}^{+}\right.$09] Shanxin Qu, Guochu Shou, Yihong Hu, Zhigang Guo, and Zongjue Qian. High throughput, pipelined implementation of aes on fpga. In Information Engineering and Electronic Commerce, 2009. IEEC'09. International Symposium on, pages 542-545. IEEE, 2009.

[RSQL04] Gaël Rouvroy, F-X Standaert, J-J Quisquater, and J-D Legat. Compact and efficient encryption/decryption module for fpga implementation of the aes rijndael very well suited for small embedded applications. In Information Technology: Coding and Computing, 2004. Proceedings. ITCC 2004. International Conference on, volume 2, pages 583-587. IEEE, 2004.

[SRHDP03] Nazar Abbas Saqib, Francisco Rodríguez-Henríquez, and Arturo DíazPérez. Aes algorithm implementation-an efficient approach for sequential and pipeline architectures. In Computer Science, 2003. ENC 2003. Proceedings of the Fourth Mexican International Conference on, pages 126-130. IEEE, 2003.

[Wu07] HongJun Wu. Hongjun's optimized C-code for AES-128 and AES-256. eSTREAM Project, 2007. http://www.ecrypt.eu.org/stream/ svn/viewcvs.cgi/ecrypt/trunk/benchmarks/aes-ctr/aes-128/ hongjun/v1/?rev=203\#dirlist.

[YGH17] Yuval Yarom, Daniel Genkin, and Nadia Heninger. Cachebleed: a timing attack on openssl constant-time rsa. Journal of Cryptographic Engineering, 7(2):99-112, 2017. 


\section{A Appendix}

\section{A.1 Round Transformation Code of Bitsliced FACE}

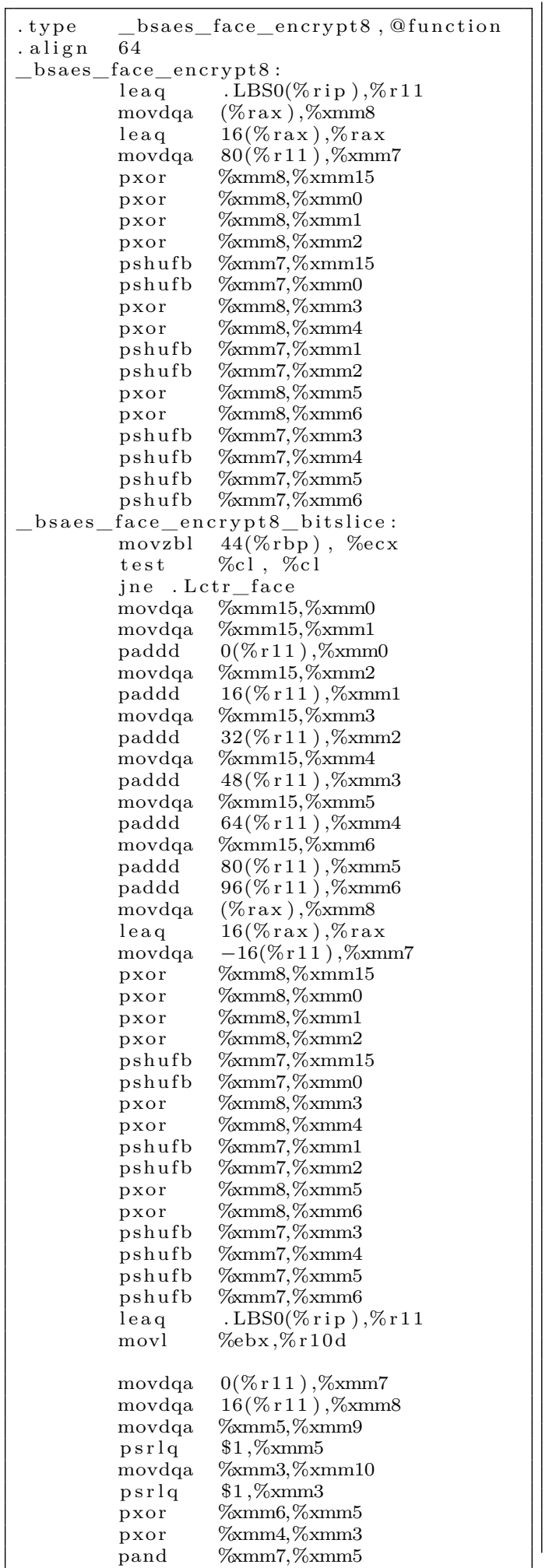

\begin{tabular}{|c|c|}
\hline and & $\% x m m 7, \% x m m 3$ \\
\hline xor & $\% \times x m m 5, \% x m m 6$ \\
\hline psllq & $\$ 1, \%$ xmm5 \\
\hline pxor & $\% x m m 3, \% x m m 4$ \\
\hline psllq & $\$ 1, \%$ xmm3 \\
\hline pxor & $\% x m m 9, \% x m m 5$ \\
\hline pxor & \%xmm10,\%xmm3 \\
\hline movdqa & \%xmm1,\%xmm9 \\
\hline psrlq & $\$ 1, \%$ xmm1 \\
\hline movdqa & $\% x m m 15, \% x m m 10$ \\
\hline srlq & $\$ 1, \% \times m m 15$ \\
\hline xor & $\% \times m m 2, \% \times m m 1$ \\
\hline xor & $\% x m m 0, \% x m m 15$ \\
\hline and & $\% \times m m 7, \% \times m m 1$ \\
\hline and & $\% \times m m 7, \% \times m m 15$ \\
\hline xor & \%xmm1,\%xmm2 \\
\hline $\mathrm{psllq}$ & $\$ 1, \% \mathrm{xmm} 1$ \\
\hline pxor & $\% \times m m 15, \% \times m m 0$ \\
\hline psllq & $\$ 1, \% \times x m m 15$ \\
\hline pxor & \%xmm9,\%xmm1 \\
\hline xor & $\% \times x m 10, \% \times m m 15$ \\
\hline lovdqa & $32(\%$ r 11$), \% \times m m$ \\
\hline ovdqa & \%xmm4,\%xmm9 \\
\hline psrlq & $\$ 2, \% \times x m m 4$ \\
\hline movdqa & \%xmm3,\%xmm10 \\
\hline psrlq & $\$ 2, \% \mathrm{xmm} 3$ \\
\hline pxor & $\% \times \mathrm{xmm} 6, \% \mathrm{xmm} 4$ \\
\hline xor & $\% \times x m 5, \% x m m 3$ \\
\hline pand & \%xmm8,\%xmm4 \\
\hline pand & \%xmm8,\%xmm3 \\
\hline xor & $\% \times x m 4, \% \times x m 6$ \\
\hline psllq & $\$ 2, \% \times \mathrm{xmm} 4$ \\
\hline pxor & $\% \times m m 3, \% \times m m 5$ \\
\hline psllq & $\$ 2, \% \times m m 3$ \\
\hline pxor & $\% x m m 9, \% x m m 4$ \\
\hline xor & $\% x m m 10, \%$ xmm3 \\
\hline ovdqa & \%xmm0,\%xmm9 \\
\hline psrlq & $\$ 2, \% \mathrm{xmm0}$ \\
\hline movdqa & $\% \times \mathrm{xm} 15, \% \mathrm{xmm} 10$ \\
\hline psrlq & $\$ 2, \% \times m m 15$ \\
\hline pxor & $\% x m m 2, \% x m m 0$ \\
\hline pxor & \%xmm1,\%xmm15 \\
\hline and & $\% x m m 8, \%$ xmm0 \\
\hline and & $\% \times x m 8, \% x m m 15$ \\
\hline xor & $\% x m m 0, \% x m m 2$ \\
\hline ps llq & $\$ 2, \% \times m m 0$ \\
\hline xor & \%xmm15,\%xmm1 \\
\hline psllq & $\$ 2, \% \mathrm{xmm} 15$ \\
\hline pxor & $\% x m m 9, \% x m m 0$ \\
\hline xor & $\% \times m m 10, \% \times m m 15$ \\
\hline movdqa & $\% \times x m 2, \% x m m 9$ \\
\hline psrlq & $\$ 4, \% \times \mathrm{xmm} 2$ \\
\hline ovdqa & \%xmm1,\%xmm10 \\
\hline psrlq & $\$ 4, \% \times \mathrm{xmm} 1$ \\
\hline pxor & $\% \times m m 6, \% \times m m 2$ \\
\hline xor & $\% \times x m 5, \% \times m m 1$ \\
\hline and & $\% \times x m 7, \% x m m 2$ \\
\hline and & $\% \times m m 7, \% x m m 1$ \\
\hline xor & $\% x m m 2, \% x m m 6$ \\
\hline $\mathrm{psllq}$ & $\$ 4, \% \times \mathrm{xmm} 2$ \\
\hline pxor & $\% \times m m 1, \% \times m m 5$ \\
\hline & $\$ 4, \% \times m m 1$ \\
\hline pxor & $\% x m m 9, \% x m m 2$ \\
\hline pxor & $\% \times x m 10, \% \times x m 1$ \\
\hline & $\% \times m m 0, \%$ xmm9 \\
\hline & $\$ 4, \% \times \mathrm{xmm0}$ \\
\hline da d & $\% \times x m 15, \% x m m 10$ \\
\hline psrlq & $\$ 4, \% \times m m 15$ \\
\hline pxor & $\% \times x m 4, \% \times m m 0$ \\
\hline & $\% \times x m 3, \% \times m m 15$ \\
\hline & $\% x m m 7, \% x m m 0$ \\
\hline & $\% x m m 7, \% x m m 15$ \\
\hline
\end{tabular}




\begin{tabular}{|c|c|c|c|}
\hline $\begin{array}{l}\text { pxor } \\
\text { psllg }\end{array}$ & $\begin{array}{l}\% \mathrm{xmm0}, \% \mathrm{xmm} 4 \\
\$ 4 \% \mathrm{xmm0}\end{array}$ & pand & $\begin{array}{l}\text { \%xmm13,\%xmm14 } \\
\text { \%xmm8,\%xmm12 }\end{array}$ \\
\hline $\begin{array}{l}\text { psllq } \\
\text { pxor }\end{array}$ & $\%$ xmm15,\%xmm3 & movdqa & $\begin{array}{l}\text { \%xmm8, \%xmm12 } \\
\% x m m 9, \% x m m 14\end{array}$ \\
\hline $\begin{array}{l}\text { pxor } \\
\text { psllq }\end{array}$ & $\$ 4, \%$ xmm15 & pxor & $\begin{array}{l}\text { \%xmm9,\%xmm14 } \\
\text { \%xmm7,\%xmm12 }\end{array}$ \\
\hline pxor & $\% \times m m 9, \% \times m m 0$ & $\begin{array}{l}\text { pxor } \\
\text { pxor }\end{array}$ & \%xmm9,\%xmm10 \\
\hline pxor & $\% x m m 10, \%$ xmm15 & pand & $\% x m m 10, \% x m m 12$ \\
\hline decl & $\%$ r10d & movdqa & \%xmm13,\%xmm9 \\
\hline decl & $\%$ r $10 \mathrm{~d}$ & pxor & \%xmm7,\%xmm12 \\
\hline & & pxor & \%xmm12,\%xmm9 \\
\hline pxor & $\% \times m m 5, \% \times m m 4$ & pxor & $\% x m m 12, \% \times x m m 8$ \\
\hline pxor & \%xmm0,\%xmm1 & pand & \%xmm7,\%xmm9 \\
\hline pxor & $\% x m m 15, \% x m m 2$ & pxor & \%xmm9,\%xmm13 \\
\hline pxor & \%xmm1,\%xmm5 & pxor & \%xmm9,\%xmm8 \\
\hline pxor & $\% x m m 15, \% x m m 4$ & pand & \%xmm14,\%xmm13 \\
\hline pxor & \%xmm2,\%xmm5 & pxor & \%xmm11,\%xmm13 \\
\hline pxor & \%xmm6,\%xmm2 & movdqa & \%xmm5,\%xmm11 \\
\hline pxor & \%xmm4,\%xmm6 & movdqa & $\% x m m 4, \% x m m 7$ \\
\hline pxor & $\% x m m 3, \% x m m 2$ & movdqa & \%xmm14,\%xmm9 \\
\hline pxor & \%xmm4,\%xmm3 & pxor & \%xmm13,\%xmm9 \\
\hline pxor & $\% \times x m 0, \% \times x m 2$ & pand & \%xmm5,\%xmm9 \\
\hline pxor & \%xmm6,\%xmm1 & pxor & $\% \times m m 4, \% \times m m 5$ \\
\hline pxor & $\% x m m 4, \% x m m 0$ & pand & \%xmm14,\%xmm4 \\
\hline movdqa & \%xmm6,\%xmm10 & pand & \%xmm13,\%xmm5 \\
\hline movdqa & \%xmm0,\%xmm9 & pxor & $\% x m m 4, \% x m m 5$ \\
\hline movdqa & $\% \times m m 4, \% x m m 8$ & pxor & \%xmm9,\%xmm4 \\
\hline movdqa & $\% \times m m 1, \% \times m m 12$ & pxor & $\% x m m 15, \% \times m m 11$ \\
\hline movdqa & $\% \times x m 5, \% \times m m 11$ & pxor & \%xmm2,\%xmm7 \\
\hline pxor & $\% \times x m 3, \% x m m 10$ & pxor & \%xmm12,\%xmm14 \\
\hline pxor & \%xmm1,\%xmm9 & pxor & \%xmm8,\%xmm13 \\
\hline pxor & $\% x m m 2, \% x m m 8$ & movdqa & $\% \times m m 14, \% \times x m 10$ \\
\hline movdqa & $\% x m m 10, \% x m m 13$ & movdqa & \%xmm12,\%xmm9 \\
\hline pxor & $\% x m m 3, \% x m m 12$ & pxor & $\% x m m 13, \% \times x m 10$ \\
\hline movdqa & \%xmm9,\%xmm7 & pxor & \%xmm8,\%xmm9 \\
\hline pxor & $\% \times m m 15, \% x m m 11$ & pand & $\% \times x m 11, \% \times x m 10$ \\
\hline movdqa & \%xmm10,\%xmm14 & pand & \%xmm15,\%xmm9 \\
\hline por & \%xmm8,\%xmm9 & pxor & \%xmm7,\%xmm11 \\
\hline por & $\% \times x m 11, \% \times x m 10$ & pxor & \%xmm2,\%xmm15 \\
\hline pxor & $\% \times m m 7, \% x m m 14$ & pand & \%xmm14,\%xmm7 \\
\hline pand & \%xmm11,\%xmm13 & pand & \%xmm12,\%xmm2 \\
\hline pxor & \%xmm8,\%xmm11 & pand & \%xmm13,\%xmm11 \\
\hline pand & $\% x m m 8, \% x m m 7$ & pand & \%xmm8,\%xmm15 \\
\hline pand & \%xmm11,\%xmm14 & pxor & \%xmm11,\%xmm7 \\
\hline movdqa & \%xmm2,\%xmm11 & pxor & \%xmm2,\%xmm15 \\
\hline pxor & $\% \times x m 15, \% \times m m 11$ & pxor & $\% \times x m 10, \% \times m m 11$ \\
\hline pand & \%xmm11,\%xmm12 & pxor & \%xmm9,\%xmm2 \\
\hline pxor & $\% x m m 12, \% \times x m 10$ & pxor & \%xmm11,\%xmm5 \\
\hline pxor & \%xmm12,\%xmm9 & pxor & \%xmm11,\%xmm15 \\
\hline movdqa & $\% \times x m 6, \% x m m 12$ & pxor & \%xmm7,\%xmm4 \\
\hline movdqa & \%xmm4,\%xmm11 & pxor & \%xmm7,\%xmm2 \\
\hline pxor & $\% \times m m 0, \% x m m 12$ & movdqa & \%xmm6,\%xmm11 \\
\hline pxor & $\% \times m m 5, \% x m m 11$ & movdqa & \%xmm0,\%xmm7 \\
\hline movdqa & $\% x m m 12, \% x m m 8$ & pxor & $\% x m m 3, \% x m m 11$ \\
\hline pand & $\% x m m 11, \% \times x m 12$ & pxor & \%xmm1,\%xmm7 \\
\hline por & \%xmm11,\%xmm8 & movdqa & $\% \times x m 14, \% \times x m 10$ \\
\hline pxor & \%xmm12,\%xmm7 & movdqa & \%xmm12,\%xmm9 \\
\hline pxor & $\% \times m m 14, \% x m m 10$ & pxor & $\% \times x m 13, \% \times m m 10$ \\
\hline pxor & \%xmm13,\%xmm9 & pxor & \%xmm8,\%xmm9 \\
\hline pxor & $\% x m m 14, \% x m m 8$ & pand & $\% x m m 11, \% x m m 10$ \\
\hline movdqa & $\% \times \mathrm{xmm} 1, \% \times \mathrm{xmm} 11$ & pand & \%xmm3,\%xmm9 \\
\hline pxor & $\% \times m m 13, \% x m m 7$ & pxor & \%xmm7,\%xmm11 \\
\hline movdqa & $\% \times x m m 3, \% \times m m 12$ & pxor & \%xmm1,\%xmm3 \\
\hline pxor & $\% x m m 13, \% x m m 8$ & pand & $\% \times m m 14, \% x m m 7$ \\
\hline movdqa & $\% x m m 0, \% x m m 13$ & pand & \%xmm12,\%xmm1 \\
\hline pand & $\% \times m m 2, \% \times m m 11$ & pand & \%xmm13,\%xmm11 \\
\hline movdqa & \%xmm6,\%xmm14 & pand & \%xmm8,\%xmm3 \\
\hline pand & $\% \times x m 15, \% \times m m 12$ & pxor & \%xmm11,\%xmm7 \\
\hline pand & \%xmm4,\%xmm13 & pxor & \%xmm1,\%xmm3 \\
\hline por & \%xmm5,\%xmm14 & pxor & \%xmm10,\%xmm11 \\
\hline pxor & $\% x m m 11, \% \times x m 10$ & pxor & \%xmm9,\%xmm1 \\
\hline pxor & $\% x m m 12, \% x m m 9$ & pxor & $\% \times x m 12, \% \times x m 14$ \\
\hline pxor & $\% x m m 13, \% x m m 8$ & pxor & \%xmm8,\%xmm13 \\
\hline pxor & $\% \times m m 14, \% x m m 7$ & movdqa & \%xmm14,\%xmm10 \\
\hline movdqa & $\% \times x m 10, \% \times x m 11$ & pxor & $\% \times m m 13, \% \times x m 10$ \\
\hline pand & \%xmm8,\%xmm10 & pand & \%xmm6,\%xmm10 \\
\hline pxor & \%xmm9,\%xmm11 & pxor & \%xmm0,\%xmm6 \\
\hline movdqa & \%xmm7,\%xmm13 & pand & \%xmm14,\%xmm0 \\
\hline movdqa & \%xmm11,\%xmm14 & pand & \%xmm13,\%xmm6 \\
\hline pxor & \%xmm10,\%xmm13 & pxor & \%xmm0,\%xmm6 \\
\hline
\end{tabular}




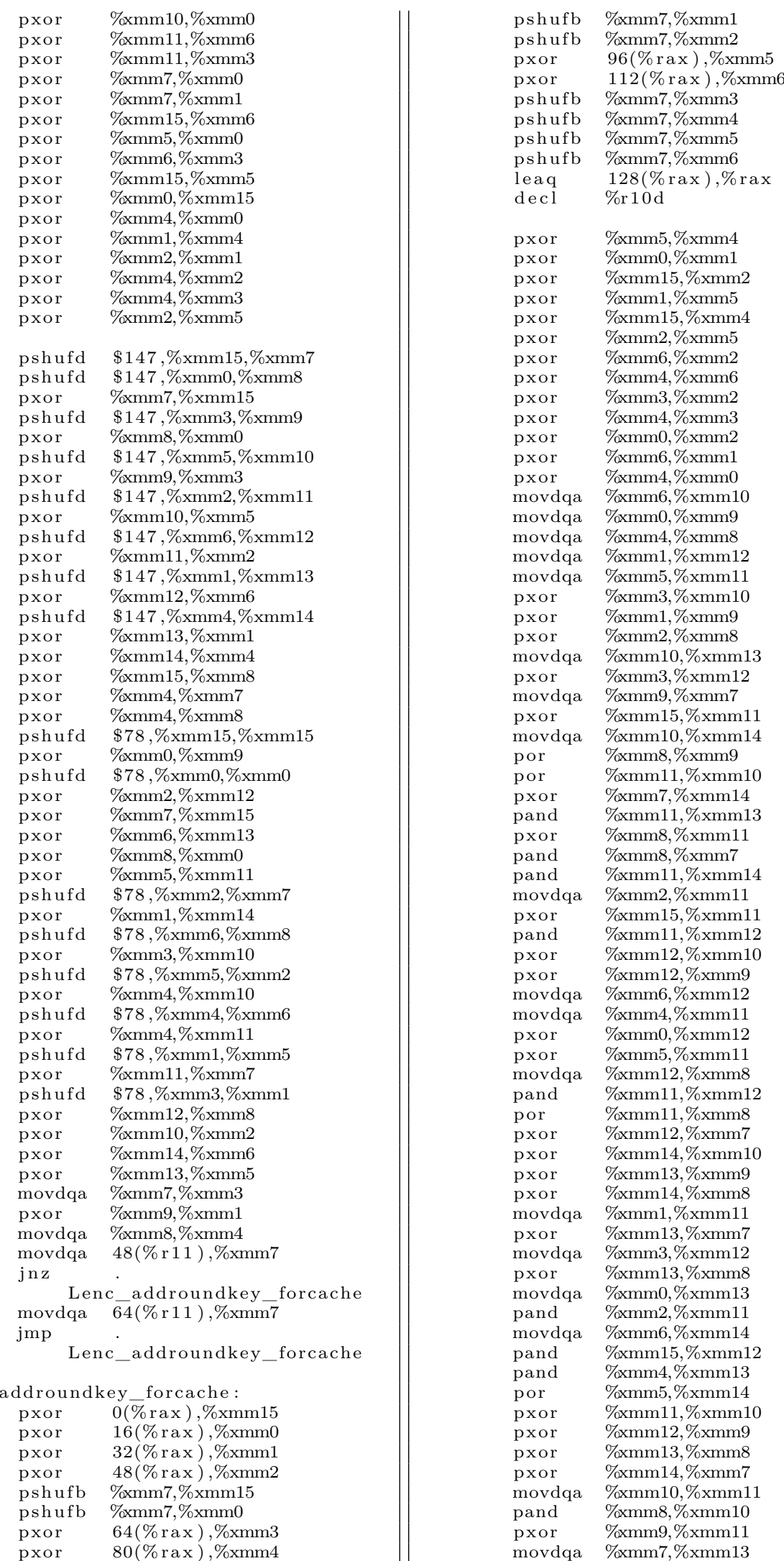




\begin{tabular}{|c|c|}
\hline qa & \\
\hline & $\% \times x m 10, \% x m n$ \\
\hline & $\% x m m 13, \% x m m 1$ \\
\hline & $\% x m m 8, \% x m m 12$ \\
\hline xor & \%xmm9,\%xmm14 \\
\hline pxor & \%xmm7,\%xmm12 \\
\hline pxor & $\% \times \mathrm{xm} 9, \% \mathrm{xmm} 10$ \\
\hline pand & $\% x \operatorname{xm} 10, \%$ xmm12 \\
\hline movdqa & $\% x m m 13, \%$ xmm9 \\
\hline pxor & $\% \times \mathrm{xm} 7, \% \mathrm{xmm} 12$ \\
\hline pxor & \\
\hline pxor & $2, \% \times m m 8$ \\
\hline pand & $\%$ xmm9 \\
\hline pxor & $\%$ xmm13 \\
\hline pxor & $\%$ xmm8 \\
\hline pand & $\% \times x m 14, \% \times x m 13$ \\
\hline pxor & $11, \% \times x m 13$ \\
\hline ovdqa & $\% \times x m 5, \% \times m m 11$ \\
\hline ovdqa & $\% \times x m m 4, \% x m m 7$ \\
\hline movdqa & \%xmm14,\%xmm9 \\
\hline pxor & \\
\hline pand & \\
\hline pxor & $\% \times r$ \\
\hline pand & $\% \mathrm{xn}$ \\
\hline pand & $6 \mathrm{xmm} 5$ \\
\hline pxor & $\% \mathrm{xr}$ \\
\hline pxor & $\% \mathrm{xr}$ \\
\hline pxor & $\mathrm{am} 11$ \\
\hline pxor & $\% \times r$ \\
\hline pxor & $\% \mathrm{xr}$ \\
\hline pxor & \\
\hline dqa & $\% x$ \\
\hline lovd & \\
\hline pxor & $\mathrm{m} 10$ \\
\hline xor & $\% \times x$ \\
\hline pand & $\mathrm{m} 10$ \\
\hline pand & $\% x$ \\
\hline pxor & $\% \times x$ \\
\hline pxor & $\% \mathrm{xI}$ \\
\hline and & $\% \times 1$ \\
\hline pand & $\% x r$ \\
\hline pand & $\mathrm{mm} 11$ \\
\hline pand & $\% \times$ \\
\hline pxor & \\
\hline pxor & \\
\hline xor & $\% \times 1$ \\
\hline pxor & $\% \times$ \\
\hline pxor & $\% x$ \\
\hline pxor & nm15 \\
\hline pxor & $\% \times$ \\
\hline pxor & \\
\hline movdqa & $\% \times \mathrm{xm}$ \\
\hline movdqa & $\% \times x m m$ \\
\hline pxor & \\
\hline pxor & $\% \times x$ \\
\hline ovdqa & $\% \mathrm{xl}$ \\
\hline lovdq & $\% \times 1$ \\
\hline pxor & $\mathrm{m} 10$ \\
\hline pxor & \\
\hline and & $\mathrm{im} 10$ \\
\hline and & $\% \times n$ \\
\hline pxor & toxmm11 \\
\hline pxor & $\% \times \mathrm{mm} 1, \% \mathrm{xmm} 3$ \\
\hline pand & \%xmm14,\%xmm7 \\
\hline pand & $\%$ oxmm1 \\
\hline & $\mathrm{xmm} 11$ \\
\hline pand & \\
\hline pxor & \\
\hline pxor & \\
\hline pxor & $\mathrm{nm} 11$ \\
\hline pxor & \\
\hline pxor & nm14 \\
\hline pxor & $\% x m m 8, \% x m m 13$ \\
\hline movdq & $\% \times x m 14, \% x m m 10$ \\
\hline pxor & $\% x m m 13, \% \times x m 10$ \\
\hline pand & \%xmm6,\%xmm10 \\
\hline & \\
\hline & \\
\hline
\end{tabular}

\begin{tabular}{|c|c|}
\hline pand & $\% x m m 13, \% x m m 6$ \\
\hline pxor & \%xmm0,\%xmm6 \\
\hline pxor & $\% x m m 10, \%$ xmm0 \\
\hline pxor & $\% x m m 11, \% x m m 6$ \\
\hline pxor & \%xmm11,\%xmm3 \\
\hline pxor & $\% x m m 7, \% x m m 0$ \\
\hline pxor & $\% x m m 7, \% x m m 1$ \\
\hline pxor & $\% x m m 15, \% x m m 6$ \\
\hline pxor & $\% \times m m 5, \% x m m 0$ \\
\hline pxor & $\% x m m 6, \% x m m 3$ \\
\hline pxor & $\% \times x m 15, \% x m m 5$ \\
\hline pxor & \%xmm0,\%xmm15 \\
\hline pxor & $\% x m m 4, \% x m m 0$ \\
\hline pxor & \%xmm1,\%xmm4 \\
\hline pxor & $\% x m m 2, \% x m m 1$ \\
\hline pxor & $\% \mathrm{xmm} 4, \% \mathrm{xmm} 2$ \\
\hline pxor & \%xmm4,\%xmm3 \\
\hline pxor & $\% \times x m 2, \% \times x m m 5$ \\
\hline pshufd & $\$ 147, \% \times x m m 15, \% \times x m 7$ \\
\hline pshufd & $\$ 147, \% \times x m m 0, \% x m m 8$ \\
\hline pxor & $\% \times x m 7, \% x m m 15$ \\
\hline pshufd & $\$ 147, \% \times x m 3, \% \times m m 9$ \\
\hline pxor & \%xmm8,\%xmm0 \\
\hline pshufd & $\$ 147, \% \times \mathrm{xmm} 5, \% \mathrm{xmm} 10$ \\
\hline pxor & $\% x m m 9, \% x m m 3$ \\
\hline pshufd & $\$ 147, \% \times \mathrm{xmm} 2, \% \mathrm{xmm} 11$ \\
\hline pxor & $\% x m m 10, \% x m m 5$ \\
\hline pshufd & $\$ 147, \% \times x m m 6, \% \times m m 12$ \\
\hline pxor & $\% x m m 11, \% x m m 2$ \\
\hline pshufd & $\$ 147, \% \times \mathrm{xmm} 1, \% \mathrm{xmm} 13$ \\
\hline pxor & $\% \mathrm{xmm} 12, \% \mathrm{xmm} 6$ \\
\hline pshufd & $\$ 147, \% \times \mathrm{xmm} 4, \% \times \mathrm{xmm} 14$ \\
\hline pxor & \%xmm13,\%xmm1 \\
\hline pxor & \%xmm14,\%xmm4 \\
\hline pxor & $\% x m m 15, \% x m m 8$ \\
\hline pxor & \%xmm4,\%xmm7 \\
\hline pxor & $\% x m m 4, \%$ xmm 8 \\
\hline pshufd & $\$ 78, \% \times \mathrm{xmm} 15, \% \mathrm{xmm} 15$ \\
\hline pxor & $\% \mathrm{xmm0, \% xmm9}$ \\
\hline pshufd & $\$ 78, \% \mathrm{xmm0}, \% \mathrm{xmm0}$ \\
\hline pxor & $\% x m m 2, \% x m m 12$ \\
\hline pxor & \%xmm7,\%xmm15 \\
\hline pxor & \%xmm6,\%xmm13 \\
\hline pxor & $\% x m m 8, \% x m m 0$ \\
\hline pxor & $\% \times \operatorname{xm} 5, \%$ xmm11 \\
\hline pshufd & $\$ 78, \% \times \mathrm{xmm} 2, \% \mathrm{xmm} 7$ \\
\hline pxor & $\% x m m 1, \% x m m 14$ \\
\hline pshufd & $\$ 78, \% \times x m m 6, \% \times m m 8$ \\
\hline pxor & $\% x m m 3, \%$ xmm10 \\
\hline pshufd & $\$ 78, \% \mathrm{xmm} 5, \% \mathrm{xmm} 2$ \\
\hline pxor & $\% x m m 4, \% x m m 10$ \\
\hline pshufd & $\$ 78, \% \times \mathrm{xmm} 4, \% \mathrm{xmm} 6$ \\
\hline pxor & $\% x m m 4, \% x m m 11$ \\
\hline pshufd & $\$ 78, \% \times x m m 1, \% \times x m 5$ \\
\hline pxor & $\% x m m 11, \% \times x m 7$ \\
\hline pshufd & $\$ 78, \% \times x m m 3, \% \times m m 1$ \\
\hline pxor & $\% x m m 12, \% x m m 8$ \\
\hline pxor & $\% \times x m 10, \% \times x m 2$ \\
\hline pxor & $\% x m m 14, \% \times x m m 6$ \\
\hline pxor & $\% x m m 13, \% x m m 5$ \\
\hline movdqa & $\% \times x m 7, \%$ xmm3 \\
\hline pxor & $\% x m m 9, \% x m m 1$ \\
\hline movdqa & $\% x m m 8, \% x m m 4$ \\
\hline movdqa & $48(\%$ r 11$), \% \times x m 7$ \\
\hline pxor & $0(\% \operatorname{rax}), \% \mathrm{xmm} 15$ \\
\hline pxor & $16(\% \mathrm{rax}), \% \mathrm{xmm0}$ \\
\hline pxor & $32(\% \mathrm{rax}), \% \mathrm{xmm} 1$ \\
\hline pxor & $48(\% \mathrm{rax}), \% \mathrm{xmm} 2$ \\
\hline pshufb & \%xmm7,\%xmm15 \\
\hline pshufb & $\% \times x m 7, \%$ xmm0 \\
\hline pxor & $64(\% \mathrm{rax}), \% \mathrm{xmm} 3$ \\
\hline pxor & $80(\% \mathrm{rax}), \% \mathrm{xmm} 4$ \\
\hline pshufb & \%xmm7,\%xmm1 \\
\hline pshufb & $\% x m m 7, \% x m m 2$ \\
\hline pxor & $96(\% \mathrm{rax}), \% \mathrm{xmm} 5$ \\
\hline
\end{tabular}


pshufb \%xmm7,\%xmm3

pshufb $\%$ xmm7,\%xmm4

pshufb \%xmm7,\%xmm5

pshufb \%xmm7,\%xmm6

leaq $128(\% \operatorname{rax}), \% \operatorname{rax}$

movdqu $248(\%$ r 15$), \% \times m m 8$

movdqa $\%$ xmm $15, \%$ xmm9

pxor $\%$ xmm8,\%xmm9

movdqu \%xmm9, 4344(\% (\% 15$)$

movdqu $264(\%$ r 15$), \% x m m 8$

movdqa $\% x m m 0, \% x m m 9$

pxor $\quad \% x m m 8, \% x m m 9$

movdqu \%xmm9, 4360(\%r15)

movdqu $280(\%$ r 15$), \%$ xmm8

movdqa $\% x m m 1, \% x m m 9$

pxor $\quad \% x m m 8, \% x m m 9$

movdqu \%xmm9, 4376(\% 15 )

movdqu 296(\% r 15$), \%$ xmm8

movdqa $\% x m m 2, \% \times m m 9$

pxor \%xmm8,\%xmm9

movdqu \%xmm9, 4392(\% r 15$)$

movdqu $312(\%$ r 15$), \%$ xmm8

movdqa $\% x m m 3, \% x m m 9$

pxor $\quad \% x m m 8, \% \times m m 9$

movdqu \%xmm9, 4408(\% r 15$)$

movdqu $328(\%$ r 15$), \% x m m 8$

movdqa \%xmm4,\%xmm9

pxor $\% x m m 8, \% x m m 9$

movdqu \%xmm9, 4424(\%r15)

movdqu $344(\%$ r 15$), \%$ xmm8

movdqa \%xmm5,\%xmm9

pxor $\%$ xmm8,\%xmm9

movdqu \%xmm9, 4440(\% 15 )

movdqu $360(\%$ r 15$), \%$ xmm8

movdqa $\% x m m 6, \%$ xmm9

pxor $\%$ xmm8,\%xmm9

movdqu $\%$ xmm9, 4456(\% (\% 15)

decl \%r10d

jmp. Lenc_sbox

. align 16
. Lctr_face :

leaq .LBSO(\% rip), \%r 11

movl \%ebx, \%r10d

leaq $248(\%$ r 15$), \%$ r 8

shl $\$ 4, \%$ ecx

add $\quad \% \mathrm{rcx}, \% \mathrm{r} 8$

leaq $\quad 4344(\%$ r 15$), \%$ r 9

movdqu (\%r9),\%xmm15

movdqu $16(\%$ r 9$), \% \times x m 0$

movdqu $32(\%$ r 9$), \% \times m m 1$

movdqu $48(\%$ r9), \% xmm2

movdqu $64(\%$ r 9$), \% x m m 3$

movdqu $80(\%$ r 9$), \% \times x m 4$

movdqu $96(\%$ r9) $\%$ \%xmm5

movdqu $112(\%$ r9), \%xmm6

movdqu (\% 8$), \% \times m m 8$

pxor $\quad \% x m m 8, \% \times m m 15$

movdqu $16(\%$ r 8$), \%$ xmm8

pxor $\quad \% x m m 8, \% x m m 0$

movdqu $32(\%$ r 8$), \%$ xmm8

pxor $\quad \% x m m 8, \% x m m 1$

movdqu $48(\%$ r 8$), \%$ xmm8

pxor $\quad \% x m m 8, \%$ xmm2

movdqu $64(\%$ r 8$), \%$ xmm8

pxor $\% x m m 8, \% x m m 3$

movdqu $80(\%$ r 8$), \% \times m m 8$

pxor $\quad \% x m m 8, \% x m m 4$

movdqu $96(\%$ r 8$), \%$ xmm8

pxor $\quad \% x m m 8, \% x m m 5$

movdqu $112(\%$ r 8$), \%$ xmm8

pxor $\quad \% x m m 8, \% \times m m 6$

leaq $272(\%$ rax $), \%$ rax

decl \%r10d

decl \%r10d

decl \%r10d

decl \%r10d

align 16

Lenc_sbox:

pxor $\quad \% x m m 5, \% x m m 4$

pxor $\quad \% x m m 0, \% \times m m 1$

pxor $\quad \% x m m 15, \% x m m 2$

pxor $\quad \% x m m 1, \% \times m m 5$

pxor $\quad \% x m m 15, \% x m m 4$

pxor $\quad \% x m m 2, \% x m m 5$

pxor $\quad \% x m m 6, \% x m m 2$

pxor $\quad \% x m m 4, \% x m m 6$

pxor $\quad \% x m m 3, \% x m m 2$

pxor $\quad \% x m m 4, \% \times m m 3$

pxor $\quad \% x m m 0, \% \times m m 2$

pxor $\quad \% x m m 6, \% x m m 1$

pxor $\quad \% x m m 4, \% x m m 0$

movdqa \%xmm6,\%xmm10

movdqa $\% x m m 0, \%$ xmm9

movdqa $\% x m m 4, \%$ xmm8

movdqa $\% x m m 1, \% x m m 12$

movdqa \%xmm5,\%xmm11

pxor $\quad \% x m m 3, \% x m m 10$

pxor $\quad \% x m m 1, \% x m m 9$

pxor $\quad \% x m m 2, \% x m m 8$

movdqa $\% x m m 10, \%$ xmm13

pxor $\quad \% x m m 3, \% x m m 12$

movdqa $\% x m m 9, \% x m m 7$

pxor $\quad \% x m m 15, \% \times m m 11$

movdqa $\% x m m 10, \% x m m 14$

por $\quad \% x m m 8, \% x m m 9$

por $\quad \% x m m 11, \% \times m m 10$

pxor $\quad \% x m m 7, \% x m m 14$

pand $\quad \% x m m 11, \% \times m m 13$

pxor $\quad \% x m m 8, \% x m m 11$

pand $\quad \% x m m 8, \% \times m m 7$

pand $\quad \% x m m 11, \% \times m m 14$

movdqa $\% x m m 2, \% x m m 11$

pxor $\quad \% x m m 15, \% \times m m 11$

pand $\quad \% x m m 11, \% \times m m 12$

pxor $\quad \% x m m 12, \% \times m m 10$

pxor $\quad \% x m m 12, \% x m m 9$

movdqa $\% x m m 6, \% \times m m 12$

movdqa $\% x m m 4, \% x m m 11$

pxor $\quad \% x m m 0, \% \times m m 12$

pxor $\quad \% x m m 5 \%$ xmm11

movdqa \%xmm12,\%xmm8

pand $\% x m m 11, \% \times m m 12$

por $\quad \% x m m 11, \% \times m m 8$

pxor $\quad \% x m m 12, \% x m m 7$

pxor $\quad \% x m m 14, \% \times m m 10$

pxor \%xmm13,\%xmm9

pxor $\quad \% x m m 14, \% x m m 8$

movdqa \%xmm1,\%xmm11

pxor $\quad \% x m m 13, \% x m m 7$

movdqa $\% x m m 3, \% \times m m 12$

pxor $\quad \% x m m 13, \% x m m 8$

movdqa $\% x m m 0, \% x m m 13$

pand $\quad \% x m m 2, \% \times m m 11$

movdqa \%xmm6,\%xmm14

pand $\quad \% x m m 15, \% \times m m 12$

pand $\quad \% x m m 4, \% \times m m 13$

por $\quad \% x m m 5, \% x m m 14$

pxor $\quad \% x m m 11, \% \times m m 10$

pxor $\quad \% x m m 12, \% x m m 9$

pxor \%xmm13,\%xmm8

pxor $\quad \% x m m 14, \% x m m 7$

movdqa \%xmm10,\%xmm11

pand $\quad \% x m m 8, \% \times m m 10$

pxor $\quad \% x m m 9, \% x m m 11$

movdqa \%xmm7,\%xmm13

movdqa \%xmm11,\%xmm14

pxor $\quad \% x m m 10, \% \times m m 13$

pand $\quad \% x m m 13 \% \times m m 14$

movdqa $\% x m m 8, \%$ xmm12

pxor $\quad \% x m m 9 \% \times m m 14$

pxor $\quad \% x m m 7, \% x m m 12$

pxor $\quad \% x m m 9, \% x m m 10$

pand $\quad \% \times m m 10 \% \times m m 12$ 


\begin{tabular}{|c|c|}
\hline movdqa & \%xmm13,\%xmm9 \\
\hline pxor & $\% \times x m 7, \% \times m m 12$ \\
\hline xor & $\% x m m 12, \% x m m 9$ \\
\hline xor & $\% \mathrm{xmm} 12, \% \mathrm{xmm} 8$ \\
\hline & \%xmm7,\%xmm9 \\
\hline xor & \%xmm9,\%xmm13 \\
\hline xor & $\% x m m 9, \% x m m 8$ \\
\hline and & $\% \times x m 14, \% \times x m 13$ \\
\hline pxor & \%xmm11,\%xmm13 \\
\hline movdqa & \%xmm5,\%xmm11 \\
\hline movdqa & \%xmm4,\%xmm7 \\
\hline movdqa & \\
\hline pxor & $3, \% \times x m 9$ \\
\hline and & \%xmm9 \\
\hline xor & \%xmm4,\%xmm5 \\
\hline and & $\% x m m 14, \% x m m 4$ \\
\hline pand & $\% x \operatorname{xm} 13, \%$ xmm5 \\
\hline pxor & \%xmm4,\%xmm5 \\
\hline pxor & $\% \times m n$ \\
\hline pxor & $\% x m m$ \\
\hline pxor & $\% \times m$ \\
\hline pxor & \\
\hline xor & $\% \times n$ \\
\hline hovdqa & $\% \times n$ \\
\hline movdq & $\% \times m$ \\
\hline pxor & $\mathrm{nm} 10$ \\
\hline pxor & $\% \times x m$ \\
\hline and & $\% x m$ \\
\hline pand & $\% \times \mathrm{xm}$ \\
\hline pxor & $\% \mathrm{xn}$ \\
\hline pxor & \\
\hline pan & \\
\hline par & \\
\hline par & $\% \mathrm{xI}$ \\
\hline par & $\% \times 1$ \\
\hline pxor & $\% \mathrm{x}$ \\
\hline pxor & 15 \\
\hline pxor & $\min 11$ \\
\hline pxor & $\% x r$ \\
\hline pxor & $\% \times n$ \\
\hline pxor & $\min 15$ \\
\hline pxor & $\% \times r$ \\
\hline pxor & $\% \times 1$ \\
\hline & $\% \times 1$ \\
\hline movdo & $\% \mathrm{xr}$ \\
\hline pxor & $\% \times r$ \\
\hline $\mathrm{p} \times 0$ & $\% \times r$ \\
\hline hovdq & $\mathrm{nm} 10$ \\
\hline movdq & \%xmm12,\%xmm9 \\
\hline pxor & $\mathrm{am} 10$ \\
\hline pxor & \\
\hline pand & L,\%xmm10 \\
\hline pand & $\% x m m 9$ \\
\hline pxor & \\
\hline pxor & $\% \mathrm{xn}$ \\
\hline & $\mathrm{m} 7$ \\
\hline D & $\% \times 1$ \\
\hline and & am11 \\
\hline pand & $\% x r$ \\
\hline pxor & $\operatorname{lm} 7$ \\
\hline pxor & n3 \\
\hline pxor & nm11 \\
\hline pxor & \\
\hline pxor & $2, \% \times \mathrm{xmm} 14$ \\
\hline pxor & $8, \%$ xmm13 \\
\hline & \\
\hline & \\
\hline & \\
\hline & $\% \times m m 0, \% \times m m 6$ \\
\hline & $\% \times x m 14, \% \times x m m 0$ \\
\hline pand & $3, \% \times m m 6$ \\
\hline pxor & \\
\hline pxor & $\% x m m 10, \%$ xmm0 \\
\hline & \%xmm11,\%xmm6 \\
\hline pxor & $\% \times m m 11, \% \times x m 3$ \\
\hline pxor & $\% \times x m 7, \% x m m 0$ \\
\hline & \%xmm7,\%xmm1 \\
\hline & 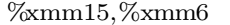 \\
\hline
\end{tabular}

$\begin{array}{ll}\text { pxor } & \text { \%xmm5,\%xmm0 } \\ \text { pxor } & \text { \%xmm6,\%xmm3 } \\ \text { pxor } & \text { \%xmm15,\%xmm5 } \\ \text { pxor } & \text { \%xmm0,\%xmm15 } \\ \text { pxor } & \text { \%xmm4,\%xmm0 } \\ \text { pxor } & \text { \%xmm1,\%xmm4 } \\ \text { pxor } & \text { \%xmm2,\%xmm1 } \\ \text { pxor } & \text { \%xmm4,\%xmm2 } \\ \text { pxor } & \text { \%xmm4,\%xmm3 } \\ \text { pxor } & \text { \%xmm2,\%xmm5 }\end{array}$

pshufd $\$ 147, \% \times m m 15, \% \times m m 7$ pshufd $\$ 147, \% \times \mathrm{xmm0}, \% \mathrm{xmm} 8$ pxor $\quad \% x m m 7, \% \times m m 15$ pshufd $\$ 147, \% \times x m 3, \% x m m 9$ pxor $\quad \% x m m 8, \% \times m m 0$

pshufd $\$ 147, \% \times x m 5, \% \times m m 10$ pxor $\quad \% x m m 9, \% \times m m 3$

pshufd $\$ 147, \% \times \mathrm{xmm} 2, \% \mathrm{xmm} 11$ pxor \%xmm10,\%xmm5

pshufd $\$ 147, \% \times \mathrm{xmm} 6, \% \times \mathrm{xmm} 12$ pxor $\quad \% x m m 11, \% \times m m 2$

pshufd $\$ 147, \%$ xmm1,\%xmm13 pxor $\quad \% x m m 12, \% x m m 6$

pshufd $\$ 147, \% \times \mathrm{xmm} 4, \% \times \mathrm{xmm} 14$

pxor $\quad \% x m m 13, \% x m m 1$

pxor $\quad \% x m m 14, \% \times m m 4$

pxor $\quad \% x m m 15, \% x m m 8$

pxor $\quad \% x m m 4, \% \times m m 7$

pxor $\quad \% x m m 4, \%$ xmm8

pshufd $\$ 78, \% \times x m 15, \% \times m m 15$

pxor $\quad \% x m m 0, \% x m m 9$

pshufd $\$ 78, \% \times x m m 0, \% \times m m 0$

pxor $\quad \% x m m 2, \% x m m 12$

pxor \%xmm7,\%xmm15

pxor $\quad \% x m m 6, \% \times m m 13$

pxor $\quad \% x m m 8, \% x m m 0$

pxor $\quad \% x m m 5, \% \times m m 11$

pshufd $\$ 78, \% \times x m 2, \% \times m m 7$

pxor $\quad \% x m m 1, \% x m m 14$

pshufd $\$ 78, \% \times x m 6, \% \times m m 8$

pxor $\quad \% x m m 3, \% \times m m 10$

pshufd $\$ 78, \% \times x m 5, \% \times m m 2$

pxor $\quad \% x m m 4, \%$ xmm10

pshufd $\$ 78, \% \times x m 4, \% \times m m 6$

pxor $\quad \% x m m 4, \% \times m m 11$

pshufd $\$ 78, \% \times x m 1, \% \times m m 5$

pxor $\quad \% x m m 11, \% \times m m 7$

pshufd $\$ 78, \% \times x m 3, \% \times m m 1$

pxor $\quad \% x m m 12, \% x m m 8$

pxor $\quad \% x m m 10, \% x m m 2$

pxor $\quad \% x m m 14, \% x m m 6$

pxor $\quad \% x m m 13, \% x m m 5$

movdqa $\% x m m 7, \%$ xmm3

pxor $\quad \% x m m 9, \% x m m 1$

movdqa $\%$ xmm8,\%xmm4

movdqa $48(\%$ r11),\%xmm7

jnz . Lenc_addroundkey

movdqa $64(\%$ r 11$), \% x m m 7$

jmp . Lenc_addroundkey

. Lenc_addroundkey :

pxor $\quad 0(\% \mathrm{rax}), \% \mathrm{xmm} 15$

pxor $\quad 16(\%$ rax $), \% x m m 0$

pxor $\quad 32(\% \operatorname{rax}), \% x m m 1$

$\begin{array}{ll}\text { pxor } & 32(\% \text { rax }), \% x m m 1 \\ \text { pxor } & 48(\% \text { rax }), \% x m m 2\end{array}$

pshufb \%xmm7,\%xmm15

pshufb \%xmm7,\%xmm0

pxor $\quad 64(\%$ rax $) \% x m m 3$

pxor $\quad 80(\%$ rax $), \% x m m 4$

pshufb \%xmm7,\%xmm1

pshufb \%xmm7,\%xmm2

pxor $\quad 96(\% \mathrm{rax}), \% \mathrm{xmm5}$

pxor $\quad 112(\%$ rax $), \% x m m 6$

pshufb \%xmm7,\%xmm3

pshufb \%xmm7,\%xmm4

pshufb \%xmm7,\%xmm5

pshufb \%xmm7,\%xmm6 


$$
\begin{array}{lr}
\text { leaq } & 128(\% \operatorname{rax}), \% \operatorname{rax} \\
\text { decl } & \text { \%r10d } \\
\text { jl } & \text {.Lenc_done } \\
\text { jmp } & \text {. Lenc_sbox }
\end{array}
$$

a lign 16

\begin{tabular}{|c|c|}
\hline pxor & $\% \times x m 9, \% \times m m 14$ \\
\hline pxor & $\% x m m 7, \% x m m 12$ \\
\hline pxor & $\% x m m 9, \% x m m 10$ \\
\hline pand & $\% \times x m 10, \% \times x m 12$ \\
\hline movdqa & $\% x m m 13, \% x m m 9$ \\
\hline pxor & $\% x m m 7, \% \times x m 12$ \\
\hline pxor & $\% x \operatorname{xm} 12, \% \times x m 9$ \\
\hline pxor & $\% x m m 12, \% x m m 8$ \\
\hline pand & \%xmm7,\%xmm9 \\
\hline pxor & $\% x m m 9, \% x m m 13$ \\
\hline pxor & $\% x m m 9, \% x m m 8$ \\
\hline pand & $\% \times x m 14, \% \times x m 13$ \\
\hline pxor & $\% \times x m 11, \% \times x m 13$ \\
\hline movdqa & \%xmm5,\%xmm11 \\
\hline movdqa & $\% x m m 4, \% x m m 7$ \\
\hline movdqa & \%xmm14,\%xmm9 \\
\hline pxor & $\% x m m 13, \% x m m 9$ \\
\hline pand & \%xmm5,\%xmm9 \\
\hline pxor & $\% \times \mathrm{xmm} 4, \% \mathrm{xmm} 5$ \\
\hline pand & \%xmm14,\%xmm4 \\
\hline pand & $\% \times m m 13, \% x m m 5$ \\
\hline pxor & $\% x m m 4, \% x m m 5$ \\
\hline pxor & \%xmm9,\%xmm4 \\
\hline pxor & $\% \times x m 15, \% \times x m 11$ \\
\hline pxor & $\% x m m 2, \% x m m 7$ \\
\hline pxor & $\% \times m m 12, \% \times x m m 14$ \\
\hline pxor & \%xmm8,\%xmm13 \\
\hline movdqa & $\% \times x m 14, \% \times x m m 10$ \\
\hline movdqa & \%xmm12,\%xmm9 \\
\hline pxor & $\% x m m 13, \% x m m 10$ \\
\hline pxor & \%xmm8,\%xmm9 \\
\hline pand & $\% x m m 11, \% x m m 10$ \\
\hline pand & \%xmm15,\%xmm9 \\
\hline pxor & \%xmm7,\%xmm11 \\
\hline pxor & $\% x m m 2, \% x m m 15$ \\
\hline pand & $\% x m m 14, \% x m m 7$ \\
\hline pand & $\% \times m m 12, \% \times m m 2$ \\
\hline pand & $\% \times x m 13, \% \times m m 11$ \\
\hline pand & $\% x m m 8, \% x m m 15$ \\
\hline pxor & $\% \times x m 11, \% x m m 7$ \\
\hline pxor & $\% \times m m 2, \% \times x m 15$ \\
\hline pxor & $\% \times x m 10, \% \times m m 11$ \\
\hline pxor & $\% x m m 9, \% x m m 2$ \\
\hline pxor & $\% x m m 11, \% x m m 5$ \\
\hline pxor & \%xmm11,\%xmm15 \\
\hline pxor & \%xmm7,\%xmm4 \\
\hline pxor & $\% \times \mathrm{xm} 7, \% \mathrm{xmm} 2$ \\
\hline movdqa & $\% x m m 6, \%$ xmm11 \\
\hline movdqa & $\% x m m 0, \% x m m 7$ \\
\hline pxor & $\% x m m 3, \% x m m 11$ \\
\hline pxor & $\% x m m 1, \% x m m 7$ \\
\hline movdqa & $\% \times x m 14, \% \times x m m 10$ \\
\hline movdqa & \%xmm12,\%xmm9 \\
\hline pxor & $\% \times x m 13, \% x m m 10$ \\
\hline pxor & $\% x m m 8, \%$ xmm9 \\
\hline pand & $\% \times x m 11, \% \times x m 10$ \\
\hline pand & $\% \times x m 3, \%$ xmm9 \\
\hline pxor & $\% x m m 7, \%$ xmm11 \\
\hline pxor & \%xmm1,\%xmm3 \\
\hline pand & $\% \times x m 14, \% x m m 7$ \\
\hline pand & \%xmm12,\%xmm1 \\
\hline pand & $\% \times x m 13, \% x m m 11$ \\
\hline pand & $\% x m m 8, \% x m m 3$ \\
\hline pxor & $\% \times x m 11, \% x m m 7$ \\
\hline pxor & $\% x m m 1, \% x m m 3$ \\
\hline pxor & $\% \times x m 10, \% \times x m 11$ \\
\hline pxor & $\% x m m 9, \% x m m 1$ \\
\hline pxor & \%xmm12,\%xmm14 \\
\hline pxor & $\% x m m 8, \%$ xmm13 \\
\hline movdqa & $\% \times m m 14, \% \times m m 10$ \\
\hline pxor & $\% \times x m 13, \% x m m 10$ \\
\hline pand & $\% x m m 6, \%$ xmm10 \\
\hline pxor & \%xmm0,\%xmm6 \\
\hline pand & $\% x \operatorname{xm} 14, \% \times x m 0$ \\
\hline pand & $\% \times m m 13, \% x m m 6$ \\
\hline pxor & \%xmm0,\%xmm6 \\
\hline pxor & $\% \times x m 10, \% x m m 0$ \\
\hline & \%xmm11 \%xmm \\
\hline
\end{tabular}

. Lenc_done:

$$
\begin{aligned}
& \text { pxor } \quad \% x m m 5, \% \times m m 4 \\
& \text { pxor } \quad \% \times m m 0, \% \times m m 1 \\
& \text { pxor } \quad \% x m m 15, \% \times m m 2 \\
& \text { pxor } \% x m m 1, \% x m m 5 \\
& \text { pxor } \quad \% x m m 15, \% x m m 4 \\
& \text { pxor } \quad \% x m m 2, \% x m m 5 \\
& \text { pxor } \quad \% x m m 6, \% \times m m 2 \\
& \text { pxor } \quad \% x m m 4, \% \times m m 6 \\
& \text { pxor } \quad \% x m m 3, \% x m m 2 \\
& \text { pxor } \quad \% x m m 4, \% x m m 3 \\
& \text { pxor } \quad \% x m m 0, \% x m m 2 \\
& \text { pxor } \quad \% x m m 6, \% x m m 1 \\
& \text { pxor } \quad \% x m m 4, \% \times m m 0 \\
& \text { movdqa \%xmm6,\%xmm10 } \\
& \text { movdqa } \% \text { xmm0,\%xmm9 } \\
& \text { movdqa } \% x m m 4, \% x m m 8 \\
& \text { movdqa } \% x m m 1, \% x m m 12 \\
& \text { movdqa } \% x m m 5, \% \times m m 11 \\
& \text { pxor } \% x m m 3, \% x m m 10 \\
& \text { pxor } \quad \% x m m 1, \% \times m m 9 \\
& \text { pxor } \quad \% x m m 2, \% \times m m 8 \\
& \text { movdqa } \% x m m 10, \% \text { xmm13 } \\
& \text { pxor } \% x m m 3, \% \times m m 12 \\
& \text { movdqa } \% x m m 9, \% x m m 7 \\
& \text { pxor } \quad \% x m m 15, \% x m m 11 \\
& \text { movdqa \%xmm10,\%xmm14 } \\
& \text { por } \quad \% x m m 8, \% \times m m 9 \\
& \text { por } \quad \% x m m 11, \% \times m m 10 \\
& \text { pxor } \quad \% x m m 7, \% \times m m 14 \\
& \text { pand } \quad \% x m m 11, \% \times m m 13 \\
& \text { pxor } \quad \% x m m 8, \% x m m 11 \\
& \text { pand } \quad \% x m m 8, \% x m m 7 \\
& \text { pand } \quad \% x m m 11, \% \times m m 14 \\
& \text { movdqa } \% \text { xmm2,\%xmm11 } \\
& \text { pxor } \quad \% x m m 15, \% x m m 11 \\
& \text { pand } \quad \% x m m 11, \% \times m m 12 \\
& \text { pxor } \quad \% x m m 12, \% x m m 10 \\
& \text { pxor } \quad \% x m m 12, \% \times m m 9 \\
& \text { movdqa \%xmm6,\%xmm12 } \\
& \text { movdqa } \% x m m 4, \% \text { xmm11 } \\
& \text { pxor } \% x m m 0, \% x m m 12 \\
& \text { pxor } \quad \% x m m 5, \% x m m 11 \\
& \text { movdqa \%xmm12,\%xmm8 } \\
& \text { pand } \quad \% x m m 11, \% \times x m 12 \\
& \text { por } \quad \% x m m 11, \% \times m m 8 \\
& \text { pxor } \quad \% x m m 12, \% x m m 7 \\
& \text { pxor } \quad \% x m m 14, \% \times m m 10 \\
& \text { pxor } \quad \% x m m 13, \% x m m 9 \\
& \text { pxor } \quad \% x m m 14, \% x m m 8 \\
& \text { movdqa } \% x m m 1, \% x m m 11 \\
& \text { pxor } \quad \% x m m 13, \% x m m 7 \\
& \text { movdqa } \% x m m 3, \% x m m 12 \\
& \text { pxor } \quad \% x m m 13, \% x m m 8 \\
& \text { movdqa } \% x m m 0, \% x m m 13 \\
& \text { pand } \% x m m 2, \% \times m m 11 \\
& \text { movdqa } \% x m m 6, \% x m m 14 \\
& \text { pand } \quad \% x m m 15, \% \times m m 12 \\
& \text { pand } \quad \% \times m m 4, \% \times m m 13 \\
& \text { por } \quad \% x m m 5, \% \times m m 14 \\
& \text { pxor } \quad \% x m m 11, \% x m m 10 \\
& \text { pxor } \quad \% x m m 12, \% x m m 9 \\
& \text { pxor \%xmm13,\%xmm8 } \\
& \text { pxor } \quad \% x m m 14, \% \times m m 7 \\
& \text { movdqa \%xmm10,\%xmm11 } \\
& \text { pand } \% \text { xmm } 8, \% \text { xmm10 } \\
& \text { pxor \%xmm9\%xmm11 } \\
& \text { movdqa } \% x m m 7, \% x m m 13 \\
& \text { movdqa \%xmm11,\%xmm14 } \\
& \text { pxor } \quad \% x m m 10, \% x m m 13 \\
& \text { pand } \quad \% x m m 13, \% \times m m 14
\end{aligned}
$$$$
\text { movdqa \%xmm8,\%xmm12 }
$$ 


\begin{tabular}{|c|c|}
\hline pxor & $\% x m m 11, \% x m m 3$ \\
\hline pxor & \%xmm7,\%xmm0 \\
\hline pxor & \%xmm7,\%xmm1 \\
\hline pxor & \%xmm15,\%xmm6 \\
\hline pxor & \%xmm5,\%xmm0 \\
\hline pxor & \%xmm6,\%xmm3 \\
\hline pxor & $\% x m m 15, \% x m m 5$ \\
\hline pxor & $\% \times m m 0, \% \times m m 15$ \\
\hline pxor & $\% x m m 4, \% x m m 0$ \\
\hline pxor & $\% \times x m 1, \% \times x m 4$ \\
\hline pxor & $\% \times m m 2, \% \times x m m 1$ \\
\hline pxor & $\% \times m m 4, \% \times x m 2$ \\
\hline pxor & \%xmm4,\%xmm3 \\
\hline pxor & $\% \times m m 2, \%$ xmm5 \\
\hline movdqa & $0(\%$ r 11$), \% \times m m 7$ \\
\hline movdqa & $16(\% \mathrm{r} 11), \% \mathrm{xmm} 8$ \\
\hline movdqa & \%xmm1,\%xmm9 \\
\hline psrlq & $\$ 1, \% \times x m m 1$ \\
\hline movdqa & $\% \times x m 2, \% x m m 10$ \\
\hline psrlq & $\$ 1, \% \times x m 2$ \\
\hline pxor & $\% \times m m 4, \% \times m m 1$ \\
\hline pxor & \%xmm6,\%xmm2 \\
\hline pand & \%xmm7,\%xmm1 \\
\hline pand & $\% x m m 7, \% \times x m 2$ \\
\hline pxor & $\% x m m 1, \% \times m m 4$ \\
\hline psllq & $\$ 1, \% \mathrm{xmm} 1$ \\
\hline pxor & \%xmm2,\%xmm6 \\
\hline psllq & $\$ 1, \% \times \mathrm{xmm} 2$ \\
\hline pxor & \%xmm9,\%xmm1 \\
\hline pxor & \%xmm10,\%xmm2 \\
\hline movdqa & $\% x m m 3, \% x m m 9$ \\
\hline psrlq & $\$ 1, \%$ xmm3 \\
\hline movdqa & $\% \times x m 15, \% \times m m 10$ \\
\hline psrlq & $\$ 1, \%$ xmm15 \\
\hline pxor & $\% x m m 5, \% x m m 3$ \\
\hline pxor & $\% \times m m 0, \% \times m m 15$ \\
\hline pand & $\% x m m 7, \% x m m 3$ \\
\hline pand & $\% x m m 7, \% x m m 15$ \\
\hline pxor & $\% x m m 3, \% x m m 5$ \\
\hline psllq & $\$ 1, \% \mathrm{xmm} 3$ \\
\hline pxor & $\% \times m m 15, \% x m m 0$ \\
\hline psllq & $\$ 1, \%$ xmm15 \\
\hline pxor & \%xmm9,\%xmm3 \\
\hline pxor & \%xmm10,\%xmm15 \\
\hline movdqa & $32(\%$ r 11$), \% \times x m 7$ \\
\hline movdqa & \%xmm6,\%xmm9 \\
\hline psrlq & $\$ 2, \% \times m m 6$ \\
\hline movdqa & $\% \times x m 2, \% \times m m 10$ \\
\hline psrlq & $\$ 2, \% \times m m 2$ \\
\hline pxor & $\% \times m m 4, \% \times m m 6$ \\
\hline pxor & $\% \times m m 1, \% \times x m m 2$ \\
\hline pand & \%xmm8,\%xmm6 \\
\hline pand & $\% x m m 8, \% \times m m 2$ \\
\hline pxor & \%xmm6,\%xmm4 \\
\hline psilq & $\$ 2, \%$ xmm6 \\
\hline pxor & $\% \times x m 2, \% \times x m 1$ \\
\hline psllq & $\$ 2, \% \times m m 2$ \\
\hline pxor & $\% x m m 9, \% x m m 6$ \\
\hline pxor & $\% \times m m 10, \% x m m 2$ \\
\hline movdqa & $\% x m m 0, \% x m m 9$ \\
\hline psrlq & $\$ 2, \% \mathrm{xmm0}$ \\
\hline
\end{tabular}

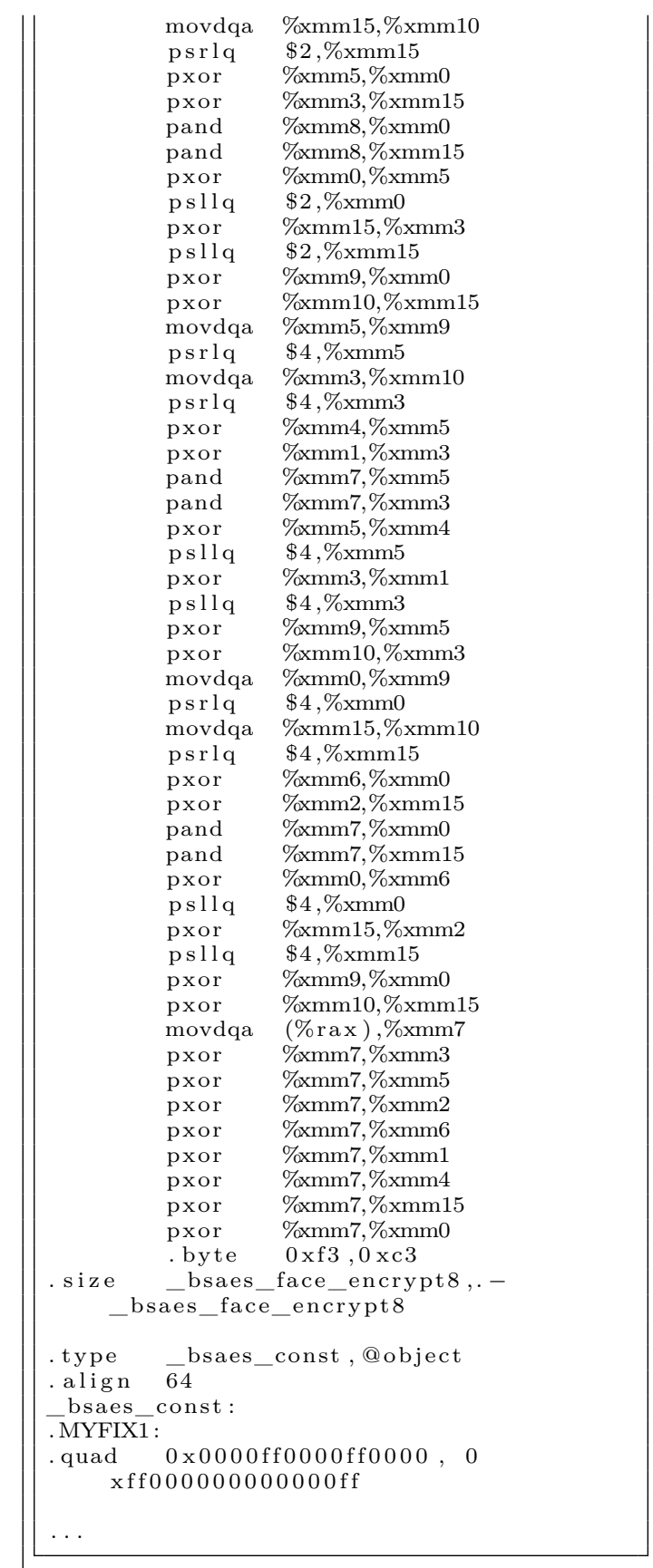

\section{A.2 Round Transformation Code of AES-NI-based FACE}

\section{A.2.1 Code for $1 \times 1$}

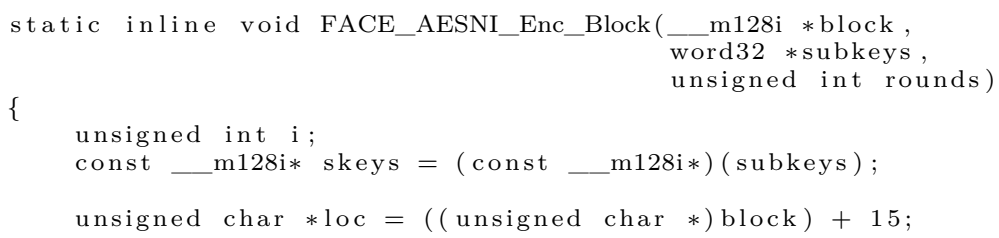




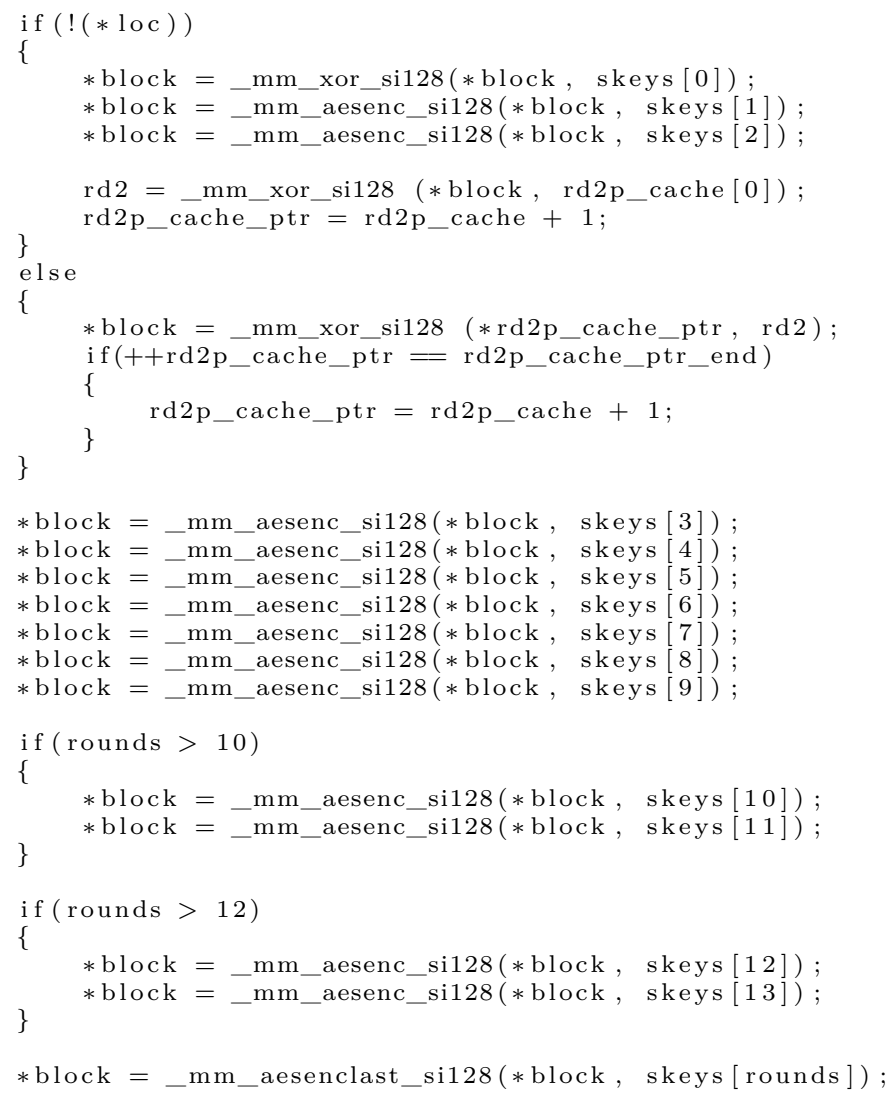

\section{A.2.2 Code for $4 \times 1$}

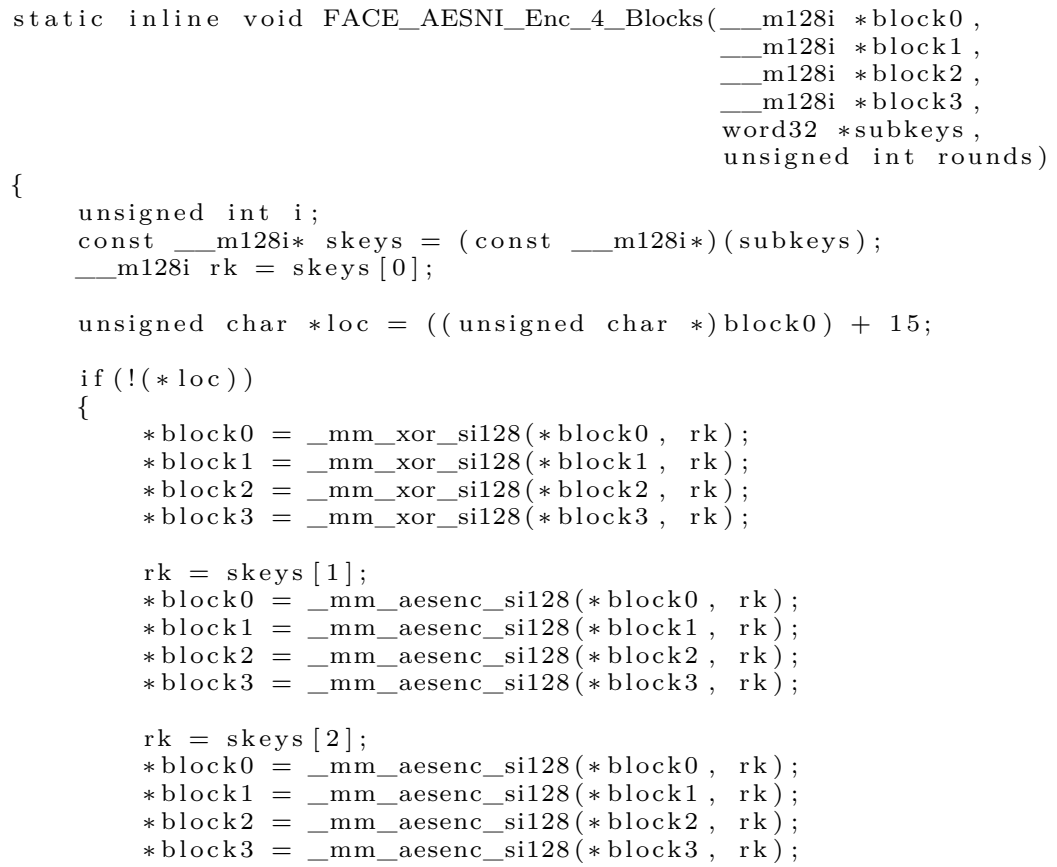




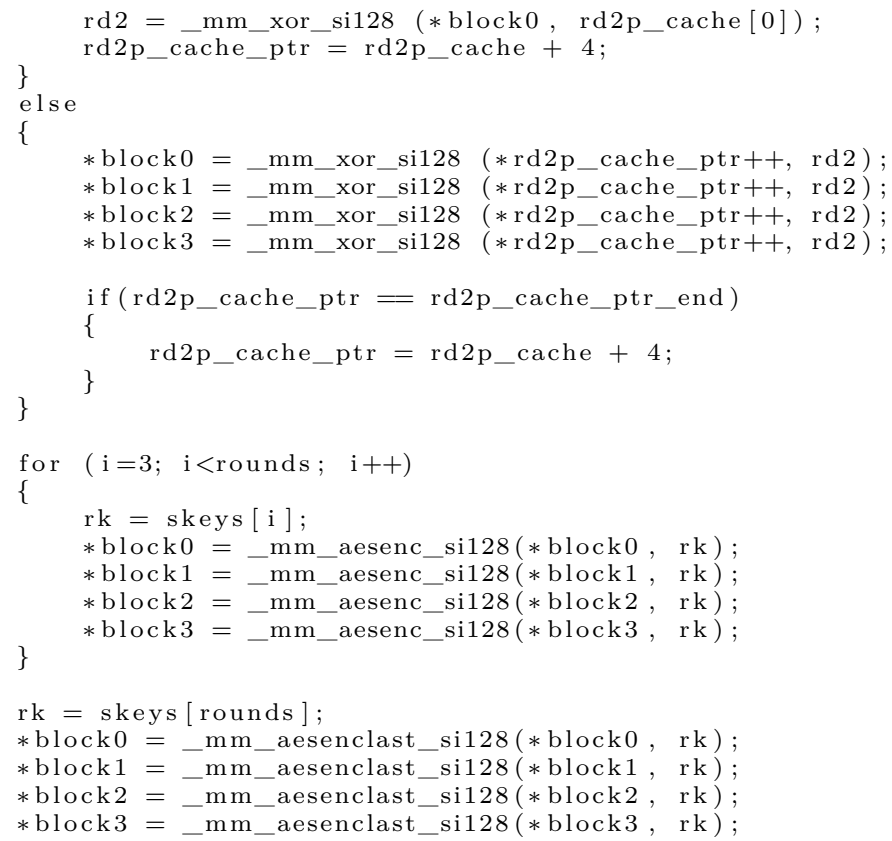

